\title{
Bandwidth and Cache Leasing in Wireless Information Centric Networks: a Game Theoretic Study
}

\author{
Michele Mangili, Student Member, IEEE, Fabio Martignon, Member, IEEE, Stefano Paris, Member, IEEE, \\ and Antonio Capone, Senior Member, IEEE
}

\begin{abstract}
Information Centric Networking (ICN) is a novel paradigm that aims at improving the performance of today's Internet by supporting caching and multicast content delivery on every network device. The main contribution of this work is to propose a centralized strategy to stimulate third parties to jointly lease the unused bandwidth and storage available on wireless access points in an ICN. We formulate this problem as a combinatorial reverse auction run by a content provider willing to increase the number of users reached by his service. We show that the optimal allocation with partial coverage problem is NP-hard, we then provide greedy heuristics that guarantee the individual rationality and truthfulness properties, and compare their performance numerically. We evaluate the benefits of our proposed mechanisms in terms of the cost savings for the content provider obtained by offloading his infrastructure through the caches, and the reduced computational time to execute the allocation algorithms. We compare the results obtained in this centralized setting, with those that can be observed when the mobile clients autonomously choose which access point they prefer to use, among those activated by the auction mechanism. We model this second scenario as a congestion game, showing that it exhibits desired properties (i.e., existence and uniqueness of a Nash Equilibrium), and comparing its social welfare with the centralized case.
\end{abstract}

Index Terms-Wireless Access Networks, Auction Theory, Information Centric Networks.

\section{INTRODUCTION}

The way customers use Internet nowadays has radically changed with respect to the original design goals that had driven the development of the TCP/IP protocol stack [1]. As a matter of fact, although Internet was initially conceived as a means to establish a point-to-point communication between a client machine and a remote server, it is currently used mostly as a content distribution infrastructure [2].

Copyright (c) 2015 IEEE. Personal use of this material is permitted. However, permission to use this material for any other purposes must be obtained from the IEEE by sending a request to pubs-permissions@ieee.org.

M. Mangili is with the Laboratoire de Recherche en Informatique (LRI), Université Paris-Sud, Paris, France, and the Dipartimento di Elettronica, Informazione e Bioingegneria (DEIB), Politecnico di Milano, Milan, Italy, (email: michele.mangili@1ri.fr).

F. Martignon is with the Laboratoire de Recherche en Informatique (LRI), Université Paris-Sud, Paris, France, and Institut Universitaire de France (IUF), (email: fabio.martignon@lri.fr).

S. Paris is with Mathematical and Algorithmic Sciences Lab, France Research Center, Huawei Technologies Co. Ltd., and with LIPADE Laboratory, Paris Descartes University, France, (email: stefano.paris@huawei.com).

A. Capone is with the Dipartimento di Elettronica, Informazione e Bioingegneria (DEIB), Politecnico di Milano, Milan, Italy, (email: antonio.capone@polimi.it).
In order to better handle this usage shift, new Information Centric Networking (ICN) design proposals for the Future Internet are recently gaining momentum. These designs have specifically been formulated to create new protocols centered around the concept of what the user is willing to retrieve, rather than where the data can be found. In fact, their distinctive characteristic is that they replace at the packet level the source and destination addresses with content names. Routing and forwarding are thus performed according to the name of the data that has to be delivered [3].

In this paper we analyze a relevant scenario where the content provider has the simultaneous objectives of (1) extending his mobile customer base by offering the users an ubiquitous access to the provided content and (2) performing server offloading by exploiting the built-in caching features of ICNs.

Since the content provider should not bear the costs of the realization of a new access network, we propose the creation of a marketplace where third party access point owners offer their unexploited bandwidth and storage resources in exchange for economic incentives for their cooperation. However, misbehaving access point owners may jeopardize the efficiency of the allocation mechanism by choosing strategically to declare false valuations for the offered resources. In order to solve this issue, auction theory provides insights for the design of tamper-proof centralized allocation mechanisms characterized by the fact that the dominant strategy of every bidder is to declare the real valuation for the provided resources.

The solution obtained with the reverse auction selects (1) which access points should be activated, (2) how much the content provider should remunerate the selected access point owners and (3) it returns the centralized solution of the mobile clients allocation to the activated access points. However, in more realistic scenarios, mobile clients are free to choose which access point their prefer to connect to, and therefore their local choice may be different than the one selected by the centralized algorithm. For this reason, we complement our work by studying also the distributed mobile clients allocation problem to the activated access points, and compare the outcome of this scenario with respect to the optimal one selected by the centralized mechanism. Therefore, we can numerically quantify how much the distributed selection of access points deviates from the centralized and ideal solution.

In our vision, the user buys a digital content, and the content provider offers the connectivity to retrieve the corresponding 
data. As an example, we believe that this "wireless shopping" business model can be effectively applied to online content stores such as e-book libraries, music and video streaming services, online magazines and newspapers as well as application stores. Some steps towards this direction have been done by Amazon, which is providing to Kindle users the "Free 3G" mobile broadband connection service to wirelessly browse the store, purchase and download the content [4].

The contributions of this paper are summarized as follows:

1) We design an optimal mechanism that can be used to motivate access point owners to jointly lease their unused bandwidth capacities and cache storage, in exchange for economic incentives. The mechanism is a reverse auction that guarantees both the individual rationality and truthfulness properties, under partial coverage constraints for the mobile clients, while forcing the access point owners to declare their real valuations for the provided resources.

2) We show that the proposed optimization problem is NP-hard. In order to cope with the computational complexity, we then provide three variants of a greedy algorithm that can be used to obtain a close to optimal solution in polynomial time, while still guaranteeing the truthfulness and individual rationality properties.

3) To further complement the previous results, while still considering the same network architecture, we take into account the case where the mobile clients autonomously connect to the activated access points, modeling their behavior as a congestion game. We prove the game is weighted potential and that the Nash Equilibrium is unique; moreover we provide a lower bound on the Price of Anarchy.

4) We provide performance comparisons of the proposed strategies. We show that all the variants of the greedy algorithm outperform the optimal one in terms of execution time. However, this comes at the cost of a reduced profit for the provider, due to the sub-optimality property of the solution computed by such greedy algorithms. Furthermore, we also compute the social welfare loss, from the optimal centralized case, with respect to the distributed scenario, where the clients autonomously connect to the access points.

This paper is structured as follows: Sec. II describes the network architecture and motivates our proposal. Sec. III formulates the optimal combinatorial reverse auction as an optimization model, while Sec. IV proposes three greedy algorithms to solve the allocation problem in polynomial time. The mobile clients' allocation problem is described in Sec. V, and extends the analysis performed in the previous sections by considering the distributed clients allocation to the available access points. Numerical results are discussed in Sec. VI, while in Sec. VII we survey related work. Finally, concluding remarks are presented in Sec. VIII.

\section{SySTEM MODEL}

In this section we illustrate the principles, definitions and assumptions characterizing the communication network of our scenario. Sec. II-A describes the network architecture and

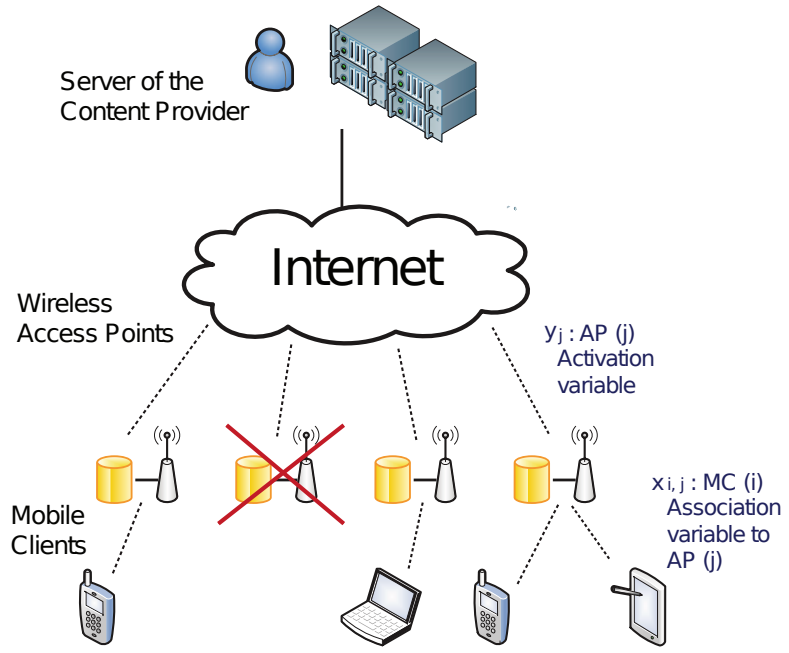

Figure 1. Network architecture. A single content provider $(C P)$ is considered in our design. Access points (APs) are equipped with caching storage. Mobile clients $(M C s)$ connect to a subset of the available APs. The demand of a $M C$ will be satisfied either by cached content, or by retrieving the data directly from the servers of the $C P$ using the available backhaul Internet connection. Our mechanism determines the MCs to APs allocation that minimizes the total cost.

discusses the benefits provided by our allocation algorithm to the content provider, while Sec. II-B clarifies the structure of the economic incentives to the access point owners and explains the properties enforced by the auction mechanism.

\section{A. Advantages for the Content Provider}

A graphical representation of the system model we consider in this paper is shown in Fig. 1. A single content provider (CP) wants to lease a set of wireless access points (APs) in order to increase his customer base by providing an ubiquitous content delivery service to mobile clients (MCs).

The core business of the provider is to distribute contents that will be accessed by the customers under payment of a fee. The hardware infrastructure owned by the CP does not comprise the radio access network, since it is beyond the scope of the service it provides; however, we assume that the $\mathrm{CP}$ owns a set of content distribution servers reachable through any Internet connection.

Raising the number of customers reached by the service jointly increases the remuneration of the $\mathrm{CP}$ as well as the operational costs (OPEX) due to the increased load of the computational infrastructure. An interesting feature offered by ICN that can mitigate the effects of this trade-off is universal caching: each AP is equipped with a variable quantity of storage that is used to memorize content previously forwarded to the destinations. For this reason, we propose that the operator leases not only the radio access and backhaul connection capacities, but also portions of the caches available at the access points. By exploiting the caching feature of ICN we make the content move across the network towards the locations where most of the users are requesting it. In our system model the backhaul Internet connection of each access point can be provided by any ISP; as a matter of fact, it will be the access point owner to choose to participate to our proposed 
bandwidth and cache sharing mechanism, in a way that is independent from the particular ISP used.

\section{B. Economic Incentives to the Access Point Owners}

Access point owners can participate to the bandwidth allocation phase by submitting to the CP the bid $\left[b_{j}, s_{j}\right]$, where $b_{j}$ is the price at which the $j$-th AP agrees to jointly share the unexploited backhaul and wireless bandwidth, as well as a quantity $s_{j}$ of cache. Our mechanism can also be applied in the scenario where Internet Service Providers actively participate to the mechanism by providing WiFi connectivity and storage; in this case, like AP owners, ISPs will submit their bids (i.e.: $\left[b_{j}, s_{j}\right]$ ). As commonly assumed in the literature (e.g., [5], [6]), in our case the real valuation $v_{j}$ of each owner is kept hidden; thus, in the most general case, $v_{j} \neq b_{j}$.

Let $p_{j} \in \mathbb{R}^{+}$be the price paid by the $\mathrm{CP}$ to lease the $j$-th AP. The utility function of the AP owner is such that:

$$
u_{j}= \begin{cases}p_{j}-v_{j} & \text { if } \mathrm{AP} j \text { is selected } \\ 0 & \text { otherwise. }\end{cases}
$$

We say that individual rationality holds if the utility of each player is always non-negative: $\forall j \in \mathcal{A}, u_{j} \geq 0$. When the access point is not selected, the owner's utility is null since he does not incur additional costs given by incoming traffic and, at the same time, he does not receive any economic incentive. Meanwhile, a rational player will choose to play only if $p_{j} \geq v_{j}$ and thus $u_{j} \geq 0$.

When the CP has collected all the sealed bids of the APs, he will select a set of access points that should be rewarded, with the aim to maximize his overall revenue. In particular, by serving the traffic demand $d_{i}$ of a mobile client $i \in \mathcal{M}$, the operator will obtain a profit $P$ per unit of bandwidth. Moreover, a cost $C$, proportional to the requests that will generate a cache miss at the access points, must also be considered, to account for the charges experienced by the CP to serve the incoming requests with his infrastructure. Thus, we would like to design a two step mechanism that 1) chooses which wireless access points should be selected among those that participated to the allocation, and 2) computes the rewards paid to the corresponding winners, in such a way that every user is forced to declare a price equal to the true valuation of his offer.

We make the assumption that each access point is directly connected to a backhaul Internet connection, whose capacity is known to the operator. We also assume that it is very unlikely that the AP owner declares a false quantity of available cache storage, since this misbehavior can be easily detected and punished by the content provider. Notwithstanding, the mechanism needs to force the AP owners to declare their real valuations, since they may be tempted to lie in order to increase their utilities by behaving strategically.

\section{Optimal Allocation And Payment Scheme}

This section illustrates the optimal mobile clients allocation and access point payment algorithm. We will derive the optimal allocation rule in terms of: 1) the prices paid by the content provider $(\mathrm{CP})$ to remunerate the selected access
Table I

SUMMARY OF THE NOTATION USED IN THIS PAPER.

\begin{tabular}{|c|c|}
\hline \multicolumn{2}{|r|}{ Parameters of the ILP model } \\
\hline $\mathcal{M}$ & Set of mobile clients \\
\hline $\mathcal{A}$ & Set of access points \\
\hline$b_{j}$ & Bid of AP $j$ \\
\hline$s_{j}$ & Storage space for caching offered by AP $j$ \\
\hline$h_{j}$ & Average hit-rate for AP $j$ \\
\hline$R_{j}$ & Backhaul bandwidth available at AP $j$ \\
\hline$L_{j}$ & Maximum coverage radius of AP $j$ \\
\hline$l_{i, j}$ & Distance between MC $i$ and AP $j$ \\
\hline$r_{i, j}$ & $\begin{array}{l}\text { Maximum Wi-Fi rate of the MC } i \text { when it is } \\
\text { connected to AP } j \text { from distance } l_{i, j}\end{array}$ \\
\hline$q_{i, j}$ & $\begin{array}{l}\text { Connectivity matrix for MC } i \text { and AP } j \text {. } \\
q_{i, j}=1 \Longleftrightarrow r_{i, j}>0, \text { otherwise } q_{i, j}=0\end{array}$ \\
\hline$d_{i}$ & Traffic demand of MC $i$ \\
\hline$C$ & $\begin{array}{l}\text { Cache miss cost per unit of bandwidth, } \\
\text { paid by the CP }\end{array}$ \\
\hline$P$ & $\begin{array}{l}\text { Average profit that a unit of bandwidth } \\
\text { generates for the } \mathrm{CP}\end{array}$ \\
\hline
\end{tabular}

\begin{tabular}{|c|l|}
\hline \multicolumn{2}{|c|}{ Variables of the ILP Model } \\
\hline$x_{i, j}$ & $\begin{array}{l}\text { Binary variable that indicates whether MC } i \text { is } \\
\text { assigned to AP } j\end{array}$ \\
\hline$y_{j}$ & $\begin{array}{l}\text { Binary variable that indicates whether AP } j \text { is } \\
\text { selected or not }\end{array}$ \\
\hline
\end{tabular}

\begin{tabular}{|l|l|}
\hline \multicolumn{2}{|c|}{ Parameters of the Auction } \\
\hline$v_{j}$ & Private valuation of AP $j$ \\
\hline$p_{j}$ & Actual price paid by the CP to the $j$-th AP owner \\
\hline$u_{j}$ & Utility function of the $j$-th AP owner \\
\hline
\end{tabular}

point owners, 2) the operational cost (OPEX) faced by the CP due to the load on his computational infrastructure, and 3) a traffic-proportional profit that the $\mathrm{CP}$ experiences for serving the clients' requests. The notation used in this paper is summarized in Table I.

We denote with $\mathcal{M}$ the set of mobile clients (MCs), while $\mathcal{A}$ is the set of access points (APs). Each MC $i \in \mathcal{M}$ generates a traffic demand $d_{i}$ that might be satisfied by at most one $\operatorname{AP} j \in \mathcal{A}$.

Due to locality constraints, the APs have a limited maximum coverage radius, that we denote with $L_{j}$ for $\mathrm{AP} j$. Let $l_{i, j}$ be the distance between MC $i$ and AP $j$, and let $r_{i, j}$ be the maximum rate of MC $i$ when connected to $\mathrm{AP} j$. If MC $i$ is beyond the range of $\mathrm{AP} j$, the corresponding rate will be null: $r_{i, j}=0, \forall i \in \mathcal{M}, j \in \mathcal{A} \mid l_{i, j}>L_{j}$. For the sake of simplicity, hereafter we assume that the rate $r_{i, j}$ refers only to the maximum possible rate between $\mathrm{MC} i$, and $\mathrm{AP} j$, regardless of the other MCs served by AP $j$. In Sec. V we extend this result by studying the behavior of the mobile clients with respect to the congestion they experience by connecting to the $\mathrm{AP} j$.

In order to participate to the auction, AP owners are required to declare $s_{j}$, the amount of storage they will offer for caching purposes. This quantity will be used by the content provider $(\mathrm{CP})$ in order to compute the average cache hit rate for $\mathrm{AP} j$, denoted by $h_{j}$. If $h_{j}=0$ this means that $\mathrm{AP} j$ does not cache any object.

Cache misses increase the load on the computational infrastructure of the CP. We denote with $C$ the cost per unit of bandwidth due to cache misses, while $P$ is the profit that 
the $\mathrm{CP}$ will experience for serving one unit of bandwidth of users' requests. Lastly, let $R_{j}$ be the bandwidth of the backhaul connection available at AP $j$.

Our formulation admits partial coverage, that is, the optimal solution may not serve all the available MCs since, in some cases, this may not be the best choice for the $\mathrm{CP}$. The binary variable $x_{i, j} \in\{0,1\}$ is used to represent the assignment between MC $i$ and AP $j$, and is such that:

$$
x_{i, j}= \begin{cases}1, & \text { if } \mathrm{MC} i \text { is assigned to } \mathrm{AP} j \\ 0, & \text { otherwise. }\end{cases}
$$

Furthermore, we denote with the binary variable $y_{j}$ the set of APs selected by the auction:

$$
y_{j}= \begin{cases}1, & \text { if AP } j \text { is selected by the auction } \\ 0, & \text { otherwise. }\end{cases}
$$

Given the above definitions and assumptions, the mobile clients allocation problem (MCAP) can be formulated as follows:

$\max P \sum_{i \in \mathcal{M}} \sum_{j \in \mathcal{A}} x_{i, j} d_{i}-\sum_{j \in \mathcal{A}} y_{j} b_{j}-\sum_{i \in \mathcal{M}} \sum_{j \in \mathcal{A}} x_{i, j} d_{i}\left(1-h_{j}\right) C$

s.t.

$$
\begin{array}{lr}
\sum_{j \in A} x_{i, j} \leq 1 & \forall i \in \mathcal{M} \\
\sum_{i \in \mathcal{M}} \frac{d_{i} x_{i, j}}{r_{i, j}} \leq 1 & \forall j \in \mathcal{A} \\
\sum_{i \in \mathcal{M}} d_{i} x_{i, j}\left(1-h_{j}\right) \leq R_{j} & \forall j \in \mathcal{A} \\
x_{i, j} \leq y_{j} & \forall i \in \mathcal{M}, j \in \mathcal{A} \\
x_{i, j} \in\{0,1\} & \forall i \in \mathcal{M}, j \in \mathcal{A} \\
y_{j} \in\{0,1\} & \forall j \in A
\end{array}
$$

The objective function (4) maximizes the total revenue of the content provider, which is given by: I) the trafficproportional profit the $\mathrm{CP}$ is going to obtain for serving MCs' demand: $P \sum_{i \in \mathcal{M}} \sum_{j \in \mathcal{A}} x_{i, j} d_{i}$; II) the incentives paid to remunerate the selected access points for their storage and access bandwidth: $\sum_{j \in \mathcal{A}} y_{j} b_{j}$; and III) the infrastructure costs caused by cache misses: $\sum_{i \in \mathcal{M}} \sum_{j \in \mathcal{A}} x_{i, j} d_{i}\left(1-h_{j}\right) C$.

It is important to note that, as in the standard Vickrey auction [7], we optimize with respect to the user's provided bids $b_{j}$ rather than the actual price paid to the AP owners. In fact, prices will be determined as a second stage, only after having selected the most promising APs. On top of that, the pricing rule that we are going to use, is such that the truthfulness property is guaranteed.

In (5) we express the set of coverage constraints, which force every mobile client to be assigned to at most one access point. Coverage is partial, because there might be cases where it is better not to serve some MCs' since it is not economically viable for the CP (e.g., if there is a single and very expensive AP).

Constraints (6) and (7) limit the number of MCs assigned to

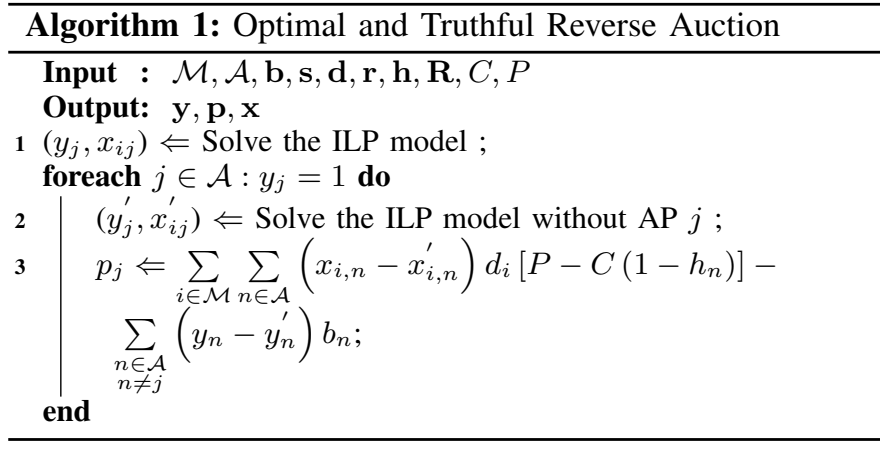

each AP, given that the radio access network and the backhaul Internet connection have a bounded capacity. Constraints (6) impose that the total demand served by an AP does not exceed the capacity of the radio access network; in particular the $i$-th MC expresses a traffic demand $d_{i}$ and is served at a rate $r_{i, j}$ when connecting to $\mathrm{AP} j$, thus consuming a fraction $\frac{d_{i}}{r_{i, j}}$ of the available wireless resources. On the other hand, constraints (7) consider the fact that the backhaul Internet connection serves only the aggregate demand that generates a cache miss (i.e., the portion of the $\mathrm{MC}$ demand that must be directly served by the CP's servers).

Constraints (8) make sure that MCs can associate only to the APs selected by the mechanism. Finally, the sets of constraints (9) and (10) express the integrality conditions on the decision variables.

After having defined the ILP model used to solve MCAP, we now illustrate the algorithm that forces the AP owners to bid their real valuations. Algorithm 1 formalizes the steps that the auctioneer should follow in order to determine the APs that should be selected as well as their payments for the provided resources. It returns the list of selected APs $\mathbf{y}$, the assignment matrix of MCs to APs $\mathbf{x}$ and the corresponding payments $\mathbf{p}$.

The algorithm proceeds in three steps. In Step 1, the ILP model (4)-(10) is solved and the maximum revenue allocation $\left(y_{j}, x_{i, j}\right)$ is computed. In Step 2, the solution of the ILP model without the $j$-th AP is determined, in other terms, the algorithm solves again the ILP model (4)-(10) with the additional constraint $y_{j}=0$. Finally, Step 3 computes the optimal price for the $j$-th AP according to the Vickrey-Clarke-Groves mechanism with Clarke pivot rule [7]. The optimal price represents the "opportunity cost" that the presence of the $j$-th AP causes to the other bidders.

Hereafter we show that the MCAP problem is NP-hard, by a polynomial time reduction of the Knapsack Problem (KP) to the ILP model (4)-(10).

Theorem 1. Complexity of the MCAP problem. The MCAP problem is NP-hard.

Proof. In order to prove the theorem we polynomial-time reduce the NP-hard Knapsack Problem (KP) [8] to MCAP.

Let $\mathcal{I}$ be a set of items of cardinality $n=|\mathcal{I}|$; for each item $i \in \mathcal{I}$, let $v_{i}$ be the value of the item and $w_{i}$ be its weight. Moreover, the binary variable $x_{i} \in\{0,1\}$ is set to 1 if item $i \in \mathcal{I}$ belongs to the subset $I \subseteq \mathcal{I}$, and 0 otherwise. The knapsack problem is to select a subset of items $I \subseteq \mathcal{I}$, such 
that their total value $\sum_{i \in \mathcal{I}} x_{i} v_{i}$ is maximized, and their total weight is bounded by $W$, that is $\sum_{i \in \mathcal{I}} x_{i} w_{i} \leq W$.

We show that every instance of the knapsack problem can be reduced in polynomial time to an instance of the mobile clients allocation problem (MCAP), as follows. First of all, we set $|\mathcal{A}|=1$, whereas $|\mathcal{M}|=n$. Furthermore, we force $h_{1}=1, b_{1}=0, d_{i}=\frac{v_{i}}{P}$ and $r_{i, 1}=\frac{v_{i} W}{P w_{i}}$. The constructed MCAP problem is as follows:

$$
\max P \sum_{i \in \mathcal{M}} \sum_{j \in \mathcal{A}} x_{i, j} \frac{v_{i}}{P}-\sum_{j \in \mathcal{A}} y_{j} 0-\sum_{i \in \mathcal{M}} \sum_{j \in \mathcal{A}} x_{i, j} d_{i}(1-1) C
$$

s.t.

$$
\begin{array}{lr}
x_{i, 1} \leq 1 & \forall i \in \mathcal{M} \\
\sum_{i \in \mathcal{M}} \frac{\frac{v_{i}}{P} x_{i, 1}}{\frac{v_{i} W}{P w_{i}}} \leq 1 & \\
\sum_{i \in \mathcal{M}} d_{i} x_{i, 1}(1-1) \leq R_{1} & \\
x_{i, 1} \leq y_{1} & \\
x_{i, j} \in\{0,1\} & \forall i \in \mathcal{M} \\
y_{j} \in\{0,1\} & \forall i \in \mathcal{M}, j \in \mathcal{A} \\
& \forall j \in A
\end{array}
$$$$
\forall i \in \mathcal{M}(12)
$$

$\forall j \in A$. (17)

The constructed instance of MCAP is equivalent to the original instance of $\mathrm{KP}$, and one such construction is done in polynomial-time. Therefore, MCAP is NP-hard.

While the standard VCG scheme is known to enforce truthfulness and individual rationality, it is defined with respect to the objective function that considers the total valuation of the players, which is given by $\sum_{j \in A} y_{j} b_{j}$. On the other hand, the MCAP objective function (4) jointly considers 1) $P \sum_{i \in M} \sum_{j \in A} x_{i, j} d_{i}$, the content provider's profit; 2$) \sum_{j \in A} y_{j} b_{j}$, the valuation of the players, and 3) $\sum_{i \in M} \sum_{j \in A} x_{i, j} d_{i}\left(1-h_{j}\right) C$, the effect of content caching.

Since the objective function we take into account is not the standard used in the VCG scheme, hereafter we prove that our mechanism jointly enforces the individual rationality and truthfulness properties.

Theorem 2. Individual Rationality. The payment rule satisfies the individual rationality property, i.e.:

$$
\forall j \in \mathcal{A} \mid u_{j} \geq 0 .
$$

Proof. Given the utility function we consider, the AP owners selected by the mechanism have a utility $u_{j}=p_{j}-v_{j}$, whereas the others experience a zero utility. Let $S W\left(y_{j}, x_{i, j}\right)$ denote the value of the social welfare (i.e.: the objective function (4)). Our payment rule for the selected APs ensures that $p_{j}=S W\left(y_{j}, x_{i, j}\right)-S W\left(y_{j}^{\prime}, x_{i, j}^{\prime}\right)+b_{j}$. Therefore, when the AP owner declares his valuation truthfully, that is $b_{j}=v_{j}$, the utility will be non-negative $u_{j} \geq 0$, since $S W\left(y_{j}, x_{i, j}\right) \geq$ $S W\left(y_{j}^{\prime}, x_{i, j}^{\prime}\right)$.

Theorem 3. Truthfulness. The payment rule ensures the truthfulness property, that is, a dominant strategy for all the players is to declare $b_{j}=v_{j}$.

Proof. Let $(x, y)$ and $(\hat{x}, \hat{y})$ be the solutions of MCAP (4)-(10), when the AP owner declares $b_{j}=v_{j}$ and $\hat{b}_{j}$, $b_{j} \neq \hat{b}_{j}$, respectively. Moreover, let $\left(x^{-j}, y^{-j}\right)$ be the solution of (4)-(10) without considering the AP $j$. The utility of $j$ when it declares $b_{j}=v_{j}$ is equal to:

$$
\begin{aligned}
u\left(b_{j}\right) & =\sum_{i \in \mathcal{M}} \sum_{n \in \mathcal{A}} x_{i, n}^{-j} d_{i}\left[C\left(1-h_{n}\right)-P\right]+\sum_{\substack{n \in \mathcal{A} \\
n \neq j}} y_{n}^{-j} b_{n}+ \\
& +\sum_{i \in \mathcal{M}} \sum_{n \in \mathcal{A}} x_{i, n} d_{i}\left[P-C\left(1-h_{n}\right)\right]-\sum_{n \in \mathcal{A}} y_{n} b_{n},
\end{aligned}
$$

While, when it declares $\hat{b}_{j} \neq v_{j}$ it has a utility equal to:

$$
\begin{aligned}
u\left(\hat{b}_{j}\right) & =\sum_{i \in \mathcal{M}} \sum_{n \in \mathcal{A}} x_{i, n}^{-j} d_{i}\left[C\left(1-h_{n}\right)-P\right]+\sum_{\substack{n \in \mathcal{A} \\
n \neq j}} y_{n}^{-j} b_{n}+ \\
& +\sum_{i \in \mathcal{M}} \sum_{n \in \mathcal{A}} \hat{x}_{i, n} d_{i}\left[P-C\left(1-h_{n}\right)\right]-\sum_{n \in \mathcal{A}} \hat{y}_{n} b_{n}+\hat{y}_{n} v_{j}-v_{j} .
\end{aligned}
$$

Given the objective function of MCAP, $(x, y)$ is the solution that maximizes (4), that is $(x, y)=$ $\operatorname{argmax} \sum_{i \in \mathcal{M}} \sum_{n \in \mathcal{A}} x_{i, n} d_{i}\left[P-C\left(1-h_{n}\right)\right]-\sum_{n \in \mathcal{A}} y_{n} b_{n}$. Moreover $v_{j}-\hat{y}_{n} v_{j} \geq 0$, in fact $\hat{y}_{n} \in\{0,1\}$. Therefore, since:

$$
\begin{aligned}
& u\left(b_{j}\right)-u\left(\hat{b}_{j}\right)=\sum_{i \in \mathcal{M}} \sum_{n \in \mathcal{A}} x_{i, n} d_{i}\left[P-C\left(1-h_{n}\right)\right]-\sum_{n \in \mathcal{A}} y_{n} b_{n}- \\
& -\left\{\sum_{i \in \mathcal{M}} \sum_{n \in \mathcal{A}} \hat{x}_{i, n} d_{i}\left[P-C\left(1-h_{n}\right)\right]-\sum_{n \in \mathcal{A}} \hat{y}_{n} b_{n}\right\}+v_{j}-\hat{y}_{n} v_{j},
\end{aligned}
$$

we have that $u\left(b_{j}\right)-u\left(\hat{b}_{j}\right) \geq 0$.

\section{Discussion}

To keep the presentation of this work as simple as possible, in our proposed formulation (4)-(10), the time dimension is not considered. We would like to remark the fact that it is however possible to add also this component to both the optimal allocation and payment scheme, as well as our proposed greedy algorithms, while preserving the individual rationality and truthfulness properties.

Since our proposed formulation fosters the partial coverage of nodes, the value of the profit parameter $P$ has a remarkable impact on the solution of the allocation scheme. As a matter of fact, by setting $P$ very small, the optimal solution for the CP will be to serve no clients at all. On the other hand, if $P$ is set large enough, the solution will serve all the clients' demands, given the available capacity of the networking infrastructure. The following proposition establishes exactly this trade-off.

Proposition 1. Using the objective function (4), the model associates the highest possible number of MCs if $P$ satisfies:

$$
P>\max _{j \in \mathcal{A}} b_{j}+C \max _{i \in \mathcal{M}} d_{i} .
$$

Proof. We show that the proposition holds, by considering the worst case: a scenario where the most expensive AP is selected, even though it does not provide any storage. 
Let $j \in \mathcal{A}$ be the access point that made the highest possible bid, and let $h_{j}=0$ be the cache hit-rate that we get at AP $j$. Let $i \in \mathcal{M}$ be the mobile client that generates the highest traffic demand among all the others (i.e., $\underset{i \in \mathcal{M}}{\operatorname{argmax}} d_{i}$ ). In the most expensive case, the $\mathrm{CP}$ activates $j$ only to serve the demand of $i$ incurring a cost $C_{\max }=\max _{j \in \mathcal{A}} b_{j}+C \max _{i \in \mathcal{M}} d_{i}$. Thus, by setting $P>C_{\max }$ we ensure that if the network has enough spare capacity, the optimal allocation rule will serve this request, since this choice will certainly lead to an improvement in the value of the objective function.

Despite its high computational cost, the VCG mechanism, which we extend in this paper, assures truthfulness, individual rationality, and social welfare maximization, which are three essential economic properties in the design of robust auctions.

Truthfulness guarantees that the dominant strategy for any bidder is to declare its private valuation $v_{j}$ for its storage space and access capacity. Therefore, the content provider can be sure of renting the cheapest APs among those that participate to the reverse auction. In contrast, in untruthful auctions bidders can manipulate the market in order to obtain higher outcomes at the expense of the content provider and other honest bidders, which might be excluded by the set of winners.

Individual rationality guarantees non-negative utilities for bidders that bid truthfully. Specifically, any AP owner that is selected by the content provider to store a portion of its data receives an amount of money at least equal to its bid, whereas any AP owner who loses the auction does not pay anything to the content provider. This property provides an incentive to participate to the reverse auction, thus maximizing the number of possible bidders.

In any auction design, the central technical problem lies in satisfying the social-efficiency, truthfulness and complexity. It is widely regarded that these properties cannot be achieved simultaneously. VCG, for example, ensures social-efficiency and truthfulness but at the price of exponential complexity. Other non-VCG truthful auctions (if there exists any, of which we are not aware), may ensure truthfulness but sacrifice socialefficiency or involve exponential computational complexity (unless we take into account stochastic auctions). Motivated by this observation, a challenging problem in auction design, as addressed in our work, is thus how to ensure truthfulness with reasonable social efficiency and complexity. Meanwhile, the designed auction mechanism should also integrate specific constraints posed by the target applications (e.g., spectrum reuse in spectrum auction design, joint band and cache leasing in our problem).

In our reverse auction settings, social welfare maximization guarantees to select the cheapest APs as winners and to minimize the content provider expenses given that all bidders bid truthfully. The overall expenses of the content provider can eventually be higher than the social welfare, since a portion of the social welfare is used as an incentive for the participation of bidders by implementing an individual rational reverse auction. Note however that, according to the revenue equivalence principle, first and second price auction yield the

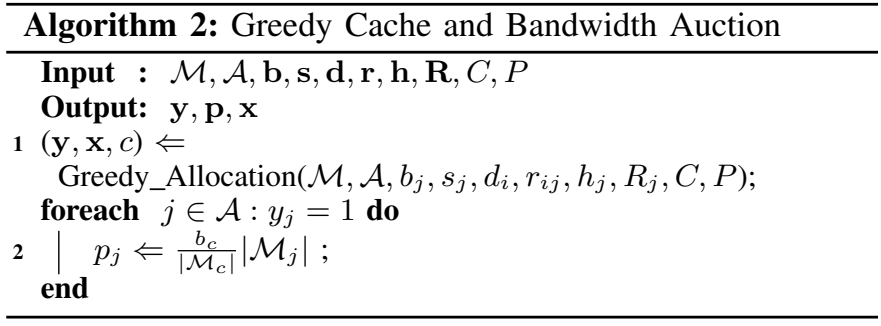

same expenses in expectation.

In fact, the revenue equivalence principle states that for any two Bayesian-Nash incentive compatible (BNIC) mechanisms, implementing the same social choice function, where the valuation of the players is drawn from a convex (or pathconnected) set, if the expected payment of player $i$ (averaged on all the private valuations of the other players) is the same for both mechanisms, then it is the same for every private valuation of the players [7]. Thus, for any BayesianNash incentive compatible (truthful) mechanisms, the expected revenue will be the same, forcing to change the social choice function to eventually reduce the overall expected "cost" perceived by the auctioneer.

\section{GReEdy Algorithms}

The computational complexity of the MCAP problem is such that, as the number of MCs $|\mathcal{M}|$ and APs $|\mathcal{A}|$ increases, the completion time of the optimal allocation (i.e., Algorithm 1) raises exponentially. In order to find an allocation strategy that can effectively scale up to large network instances, this section provides a computationally efficient, polynomial-time algorithm with three alternative strategies, that still guarantees truthfulness and individual rationality. We begin by describing the greedy $M C$ alternative, while we will talk about the other greedy strategies at the end of the section.

Let $k_{j}=\frac{b_{j}}{\left|\mathcal{M}_{j}\right|}$ be the ratio between the bid of the $j$-th $\mathrm{AP}\left(b_{j}\right)$ and the number of MCs that it can potentially serve, $\left|\mathcal{M}_{j}\right|$, given the radio access coverage constraints only. The quantity $k_{j}$ can be interpreted as the price per mobile client that should be paid to the $j$-th AP if it served all the MCs in its range. Let $c$ be the index of the critical access point, which is defined as the first AP that has not been selected by the mechanism. The input values shared by the algorithms (i.e., $\mathcal{M}, \mathcal{A}, \mathbf{b}, \mathbf{s}, \mathbf{d}, \mathbf{r}, \mathbf{h}, \mathbf{R}, C, P)$, are the same parameters we used to characterize the optimal allocation problem as in Table I.

In the same way as in the optimal auction, the greedy algorithm (Algorithm 2) is characterized by the following two phases: 1) the allocation phase, which selects the APs characterized by the lowest $k_{j}=\frac{b_{j}}{\left|\mathcal{M}_{j}\right|}$ until the demands of MCs are satisfied (Step 1, Alg. 2), and 2) the payment phase, which determines the price paid to the selected APs, given the characteristics of the critical access point $c$ (Step 2, Alg. 2).

The greedy allocation of MCs to APs, as well as the identification of the critical access point $c$, are performed by Algorithm 3. The allocation procedure (Alg. 3) starts by removing the non-profitable APs (Step 1), and sorts 

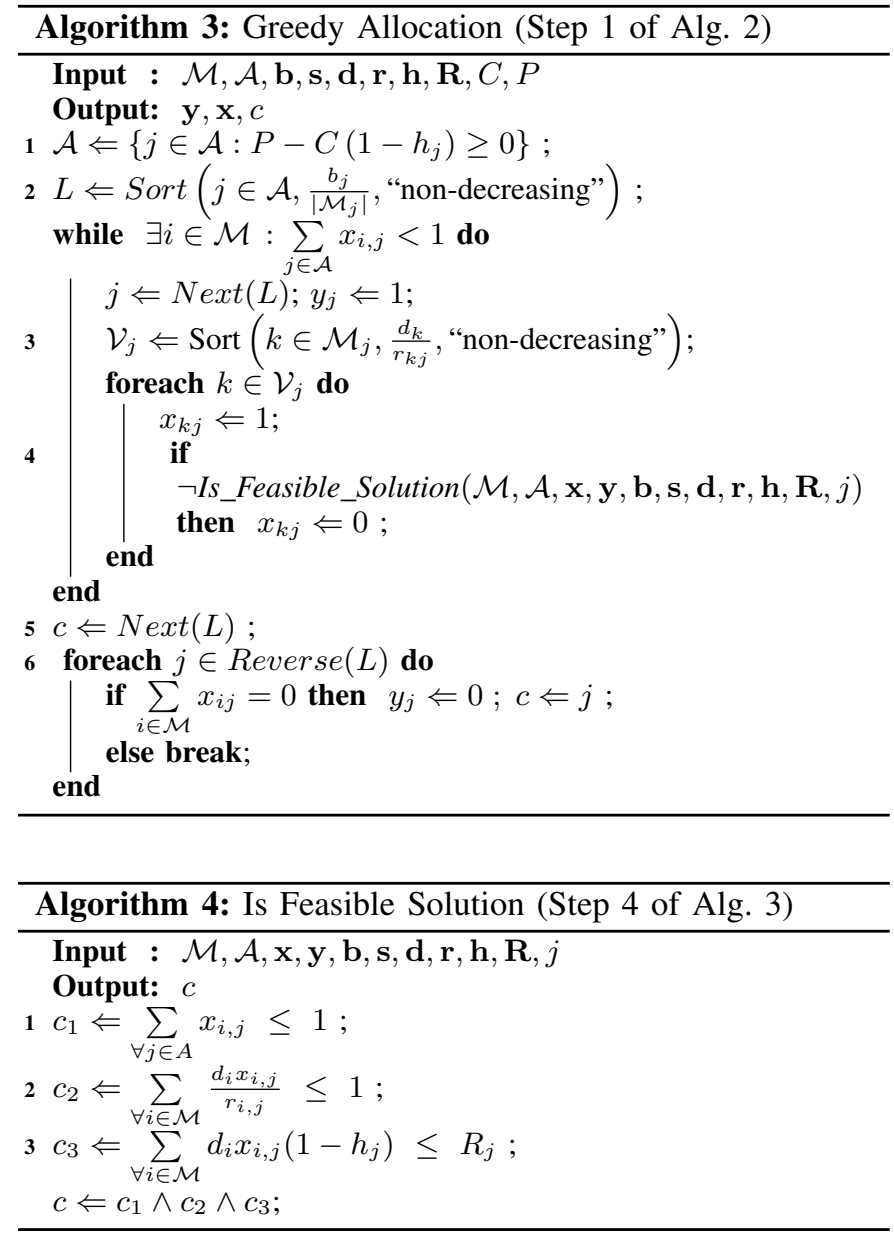

the APs in non-decreasing order according to the corresponding $k_{j}$ values (Step 2). This is done with the aim of selecting the "most promising" APs in terms of the declared price per number of reachable mobile clients. We then iteratively allocate to the APs the largest number of unassigned mobile clients by choosing those which have lower capacity demand $\frac{d_{i}}{r_{i j}}$ (Step 3), while still preserving the feasibility of the solution (Step 4). Before completing the execution of Alg. 3, in Steps 5 and 6 we set the value of the critical access point, which is the first AP that has not been allocated any MC by the algorithm (the first looser).

Finally, Algorithm 4 checks if the solution is feasible, by determining whether the constraints of the ILP model hold. In detail, Alg. 4 checks in Step 1 if each MC is assigned to at most one AP, as imposed by constraints (5); Step 2 checks whether the available Wi-Fi bandwidth is not saturated, as in constraints (6), and lastly Step 3 checks if the backhaul connection can accommodate the allocated traffic, as in constraints (7).

Alg. 2 implements a truthful auction since 1) the allocation phase respects the monotonicity property as the APs are sorted in non-increasing order of their bids per number of covered mobile customers, and 2) there exists a critical value which determines whether the AP has been selected or not. As demonstrated in [7] the previous two conditions ensure the truthfulness of the greedy algorithm.
Table II

SUMMARY OF THE 3 PROPOSED VARIANTS FOR THE GREEDY ALGORITHM

\begin{tabular}{|l|l|l|}
\hline $\begin{array}{l}\text { Greedy } \\
\text { Variant }\end{array}$ & Step 2, Alg. 2 & Step 2, Alg. 3 \\
\hline $\begin{array}{l}\text { MC } \\
\text { (G.m.) }\end{array}$ & $p_{j} \Leftarrow \frac{b_{c}}{\left|\mathcal{M}_{c}\right|}\left|\mathcal{M}_{j}\right|$ & $L \Leftarrow \operatorname{Sort}\left(j \in \mathcal{A}, \frac{b_{j}}{\left|\mathcal{M}_{j}\right|}\right.$, "non-decr") \\
\hline $\begin{array}{l}\text { Cache } \\
\text { (G.c.) }\end{array}$ & $p_{j} \Leftarrow \frac{b_{c}}{h_{c}} h_{j}$ & $L \Leftarrow \operatorname{Sort}\left(j \in \mathcal{A}, \frac{b_{j}}{h_{j}}\right.$, "non-decr") \\
\hline $\begin{array}{l}\text { Backhaul } \\
\text { (G.b.) }\end{array}$ & $p_{j} \Leftarrow \frac{b_{c}}{R_{c}} R_{j}$ & $L \Leftarrow \operatorname{Sort}\left(j \in \mathcal{A}, \frac{b_{j}}{R_{j}}\right.$, "non-decr") \\
\hline
\end{tabular}

By jointly substituting Step 2 of Alg. 2 and Step 2 of Alg. 3, as detailed in Table II, we can change the optimization strategy of the greedy algorithm; in particular, we propose the following three variants:

1) Greedy $M C$ (G.m.);

2) Greedy cache (G.c.);

3) Greedy backhaul (G.b.).

As discussed previously, the greedy $M C$ (G.m.) alternative gives higher priority to the APs that can potentially serve more MCs, due to their space location. On the other hand, the greedy cache (G.c.) selects the APs that are leasing larger caching storage; lastly, the greedy backhaul (G.b.) chooses the APs with a faster backhaul Internet connection. Table II summarizes these three variants, which will be compared numerically in Sec. VI. Finally, we observe that all the variants we propose do not modify the monotonicity property of the allocation phase; therefore, it is straightforward to show that truthfulness and individual rationality still hold.

\section{A. Greedy Algorithm Analysis}

In this section we analyze the greedy algorithm: we discuss its computational complexity, then we compare its efficiency with the optimum, in terms of both Social Welfare maximization and auction payments. In particular, we show that under the same conditions that make the greedy select a solution that leads to an inefficient Social Welfare, such solution will still be economically competitive in terms of auction payments.

First of all, we observe that assuming the number of mobile clients is much larger than the access points involved in the allocation (i.e.: $|\mathcal{M}| \gg|\mathcal{A}|$ ), then the worst-case complexity of the greedy algorithm is quadratic with the number of MCs (i.e.: $O\left(|\mathcal{M}|^{2}\right)$ ).

To show the efficiency of the solution computed by the greedy, we focus, for the sake of simplicity, on the Greedy backhaul (G.b.) variant of the algorithm, even though the same key conclusions can be obtained also for the other formulations. We consider an instance where two access points $\mathcal{A}=\{1,2\}$ (AP1 and AP2) can serve a mobile client (MC). To keep the presentation simple, we assume that the content catalog can be entirely cached in both nodes, leading to a hitrate of $100 \%$ (i.e.: $h_{1}=h_{2}=1$ ), while the profit per unit of demand is $P$ (with $P \gg 1$ ). Let the mobile client's demand be $d_{1}=1$ and the rates on the wireless links be $r_{1,1}=r_{1,2}=1$. Assume that the APs' bids are $b_{1}=1+\epsilon(\epsilon>0$ and close to zero) and $b_{2}=D$ (with $D>1$ ), while the AP's available backhaul bandwidth is $R_{1}=\frac{1}{D}$ and $R_{2}=1$. 
In this scenario, the optimal allocation forces the $\mathrm{MC}$ to connect to AP1 since its bid is lower than the one of AP2, and the social welfare (4) is $S W^{o}=P-(1+\epsilon)$. On the other hand, if we consider the greedy algorithm, it forces the MC to connect to the AP2 (since $\frac{b_{2}}{R_{2}}<\frac{b_{1}}{R_{1}}$ ), leading to a social welfare of $S W^{g}=P-D$. We can therefore observe that the Social Welfare Ratio $\left(S W_{\text {Ratio }}=\frac{S W^{g}}{S W^{\circ}}\right)$ between the greedy and the optimal solution tends to $-\infty$ when the bid $D$ is very large:

$$
S W_{\text {Ratio }}=\frac{S W^{g}}{S W^{o}}=\lim _{D \rightarrow+\infty} \frac{P-D}{P-(1+\epsilon)}=-\infty .
$$

Therefore, as shown in this scenario, the greedy algorithm can reach very unfavorable solutions in terms of Social Welfare. In other words, the greedy scheme does not guarantee the maximization of the social welfare. Instead, it might lead to a transfer of utility from the content provider to the AP owner.

At the same time, if we focus on the actual payments that both the allocation mechanisms select, we observe that the greedy algorithm leads to a close to optimum solution. In order to perform one such analysis, we take into account the Offloading Cost Ratio (OCR), which we define as the ratio between the actual payment of the greedy algorithm and the one of the optimal allocation:

$$
O C R=\frac{p^{g}(x, y)}{p^{o}(x, y)}=\frac{\sum_{j \in \mathcal{A}} p_{j}^{g}}{\sum_{j \in \mathcal{A}} p_{j}^{o}}=\lim _{D \rightarrow+\infty} \frac{(1+\epsilon) D}{D}=(1+\epsilon),
$$

where $p_{j}^{g}$ and $p_{j}^{o}$ denote the price paid by AP $j$ according to the greedy and the optimal algorithm, respectively. In other words, the metric that mostly interests network operators (i.e.: the OCR, or the overall payment they need to make in order to reserve the resources they booked) is such that the payment required by the greedy algorithm is only infinitesimally larger than the one computed for the optimal allocation, even though in terms of social welfare, the solution obtained with the greedy algorithm is not efficient.

It is important to note that the conditions we imposed in the example above are hardly met in a real scenario, since operators do not provide backhaul bandwidth with such a small granularity. Indeed, when $D \rightarrow+\infty$, the available backhaul bandwidth for AP 1 tends to zero $\left(R_{1}=\frac{1}{\infty}=0^{+}\right)$, which can hardly be satisfied by any network operator. Therefore, a consequence of this observation is that we can bound the $S W_{\text {Ratio }}$ by fixing the minimum amount of bandwidth any access point owner should provide in order to participate to the mechanism.

\section{Network Selection Game}

Our proposed optimal allocation strategy and the corresponding heuristic algorithms are formulated for a centralized setting in which the content provider (CP) optimally allocates the mobile clients (MCs) to the access points (APs). However, in a real scenario, $\mathrm{MC}$ owners typically choose autonomously which access point their devices should connect to. At the same time, each user's choice will influence the others: as a matter of fact, by changing the AP at which a given $\mathrm{MC}$ is connected, the MC owner is indeed affecting the quality of the wireless channel perceived by the other users.

In this scenario, game theory is the natural tool used to model the players' mutual interaction (e.g., [9], [10]). In

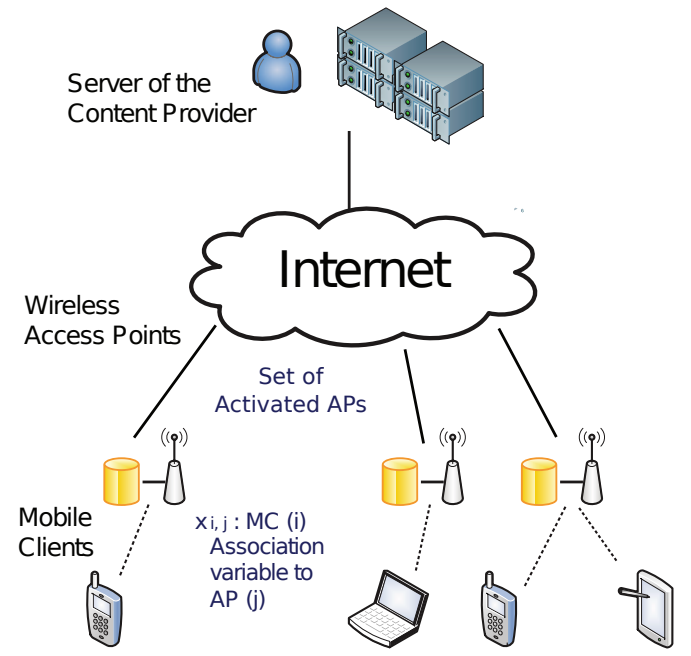

Figure 2. Network Selection Game Architecture. The architecture we consider in the Network Selection Game is very similar to the one we took into consideration in the previous Sections II-IV. However, while previously we assumed that both the access point activation and the mobile clients association to the access point were optimally chosen by a centralized entity, in the network selection game, we instead consider the case when mobile clients autonomously select (and compete) which access point they prefer to use.

particular, in this section we consider the same network architecture analyzed so far, but we extend our analysis by taking into account the fully distributed case where mobile clients autonomously choose which access point they prefer to use. Fig. 2 shows the architecture we take into consideration in this section of the manuscript. We model this practical scenario as a non-cooperative network selection game in which the players (i.e., the MCs) interact with each other by selfishly selecting the access network (i.e., the AP) that minimizes their cost. We chose to provide this analysis in our paper in order to 1) derive the performance loss that the non-cooperative, distributed allocation causes, compared to the centralized global optimum and 2) complement our contribution by considering also the case when MCs autonomously select to which AP they should connect.

In this scenario, a mobile client $i \in \mathcal{M}$ can be served by multiple APs in its current location. Different APs will likely provide different utilities to the players, and, at the same time, the utility will be a function of the other players' choices. In such distributed setting, all the MCs that can be served by at least one AP will connect to the wireless infrastructure. On the other hand, all the mobile clients that are not covered by the signal of an activated access point will not participate to the network selection game.

For the sake of simplicity, hereafter we denote with $\mathcal{A}$ the set of activated access points, and with $\mathcal{M}$ the set of mobile clients that can be reached by at least one AP. The quality metric that we consider relevant for the MCs is the congestion level experienced while connecting to a specific AP. In particular, due to the well known 802.11 anomaly [11], we assume that the rate experienced by all the MCs connected to an AP $j \in \mathcal{A}$ is the same and it is equal to $r_{j}=\min _{i \in \mathcal{M} \mid l_{i, j} \leq L_{j}} r_{i, j}$, that is the rate of the farthest MC potentially served by the AP. We therefore define the cost function of player $i \in \mathcal{M}$ as 
follows: $c_{i}(x)=\sum_{\forall k \in M, \forall j \in A} \frac{x_{i, j} \cdot x_{k, j}}{r_{j}}$.

Let $S=\mathrm{x}$ be a strategy profile. The choice of player $i \in \mathcal{M}$ is represented with $\mathbf{x}_{i}$, while the one of the other players is $\mathbf{x}_{-i}$. In particular, the binary variable $x_{i, j}=1$ if and only if MC $i$ is connected to AP $j$. The solution concept we adopt is the pure strategy Nash Equilibrium (NE): a strategy profile $S$ in which there is no player that can improve its utility by unilaterally deviating from his choice.

In this section we prove that the network selection game is weighted potential, and therefore by letting players play the best response dynamics, one Nash equilibrium will be reached. Furthermore, we also prove that there exists only one such Nash Equilibrium.

The rationale behind the following proofs is as follows: consider the mobile client $i \in \mathcal{M}$, and assume that it is currently connected to the AP $j \in \mathcal{A}$. The cost (i.e., the congestion) perceived by such mobile client is $c_{i}(x)=\sum_{\forall k \in \mathcal{M}, \forall j \in \mathcal{A}} \frac{x_{i, j} \cdot x_{k, j}}{r_{j}}=$ $\sum_{i \in \mathcal{M}} \frac{x_{i, j}}{r_{j}}$. A Nash Equilibrium is a strategy profile such that if MC $i$ migrates to another AP $k \neq j$, it should observe a higher cost (i.e.: $\frac{1}{r_{k}}+\sum_{i \in \mathcal{M}} \frac{x_{i, k}}{r_{k}}>\sum_{i \in \mathcal{M}} \frac{x_{i, j}}{r_{j}}, \forall k \in \mathcal{M}, k \neq i$ ).

Hereafter we show that the network selection game is weighted potential, and therefore the distributed solution obtained with the best response dynamics converges to a $\mathrm{NE}$ [12].

Proposition 2. Weighted Potential: Our proposed network selection game is weighted potential with potential function $\Phi(\mathbf{x})=\sum_{k \in \mathcal{M}} \sum_{j \in \mathcal{A}} \sum_{i \in \mathcal{M}} \frac{x_{k, j} x_{i, j}}{r_{j}}+\sum_{j \in \mathcal{A}} \frac{1}{r_{j}} \sum_{i \in \mathcal{M}} x_{i, j}$.

Proof. To prove that our proposed game is weighted potential, we must show that, given the strategy space $\mathcal{S}$ there is a potential function $\Phi: \mathcal{S} \rightarrow \mathbb{R}$ and a vector $\mathbf{w} \in \mathbb{R}_{+}^{|\mathcal{M}|}$ such that $\forall \mathbf{x}_{-i} \in \mathcal{S}_{-i}, \forall \mathbf{x}_{i}, \mathbf{x}_{i}^{\prime} \in \mathcal{S}_{i}$ it holds that

$$
\Phi\left(\mathbf{x}_{i}, \mathbf{x}_{-i}\right)-\Phi\left(\mathbf{x}_{i}^{\prime}, \mathbf{x}_{-i}\right)=w_{i}\left(u_{i}\left(\mathbf{x}_{i}, \mathbf{x}_{-i}\right)-u_{i}\left(\mathbf{x}_{i}^{\prime}, \mathbf{x}_{-i}\right)\right)
$$

Consider the AP $i \in \mathcal{A}$. Let $x_{i, j}=\left\{\begin{array}{ll}1 & \text { if } j=h \\ 0 & \text { otherwise }\end{array}\right.$, while $x_{i, j}^{\prime}=\left\{\begin{array}{ll}1 & \text { if } j=q \\ 0 & \text { otherwise }\end{array}\right.$.

In other words, $\mathbf{x}_{i}$ states that $\mathrm{MC} i$ is connected to $\mathrm{AP} h$, whereas $\mathbf{x}_{i}^{\prime}$ states that MC $i$ is connected to AP $q$. Let $n_{i, j}=$ $\sum x_{w, j}$ be the number of MCs, excluding $i$, connected $w \in \mathcal{M} \backslash\{i\}$

to $\operatorname{AP} j$. Then we have:

$$
u_{i}\left(\mathbf{x}_{i}, \mathbf{x}_{-i}\right)-u_{i}\left(\mathbf{x}_{i}^{\prime}, \mathbf{x}_{-i}\right)=\frac{r_{q}+r_{q} n_{i, h}-r_{h}-r_{h} n_{i, q}}{r_{h} r_{q}} .
$$

Moreover, considering the potential function we obtain:

$$
\begin{aligned}
& \Phi\left(\mathbf{x}_{i}, \mathbf{x}_{-i}\right)-\Phi\left(\mathbf{x}_{i}^{\prime}, \mathbf{x}_{-i}\right)= \\
& =\frac{\left(n_{i, h}+1\right)^{2}+\left(n_{i, h}+1\right)}{r_{h}}+\frac{\left(n_{i, q}\right)^{2}+n_{i, q}}{r_{q}} \\
& -\frac{\left(n_{i, h}\right)^{2}+n_{i, h}}{r_{h}}-\frac{\left(n_{i, q}+1\right)^{2}+\left(n_{i, q}+1\right)}{r_{q}}= \\
& =2\left(\frac{r_{q}+r_{q} n_{i, h}-r_{h}-r_{h} n_{i, q}}{r_{h} r_{q}}\right),
\end{aligned}
$$

and therefore the game is weighted potential since it is sufficient to set $\forall i \in \mathcal{A}, w_{i}=2$.

The existence of a Nash Equilibrium is guaranteed by the previous Proposition; we now formally prove that there exists only one Nash Equilibrium. In particular, the following Theorem proves that in our proposed network selection game, Nash Equilibria are "essentially unique" [7], meaning that all equilibrium configurations have the same overall cost.

Theorem 4. Uniqueness of the Nash Equilibrium: Our proposed network selection game has an essentially unique Nash Equilibrium.

Proof. Let $n_{j}=\sum_{k \in \mathcal{M}} x_{i, j}$ be the number of users simultaneously connected to the AP $j \in \mathcal{A}$; for the sake of clarity, we can then rewrite the potential function as follows:

$$
\begin{array}{r}
\Phi(\mathbf{x})=\sum_{k \in \mathcal{M}} \sum_{j \in \mathcal{A}} \sum_{i \in \mathcal{M}} \frac{x_{k, j} x_{i, j}}{r_{j}}+\sum_{j \in \mathcal{A}} \frac{1}{r_{j}} \sum_{i \in \mathcal{M}} x_{i, j}= \\
=\sum_{j \in \mathcal{A}} \frac{n_{j}^{2}}{r_{j}}+\sum_{j \in \mathcal{A}} \frac{n_{j}}{r_{j}}=\Phi(\mathbf{n}) .
\end{array}
$$

By the definition of the potential function itself [7], Nash Equilibria can be computed by solving the optimization problem: $\min \Phi(\mathbf{n})$, subject to $\sum_{j \in \mathcal{A}} n_{j}=|\mathcal{A}|$. One such optimization problem is a convex Mixed-Integer Non-Linear Program, in fact, the continuous relaxation of the objective function is convex.

For this class of problems, a local optimal solution is also equal to the global optimum [13], [14], thus the Nash Equilibrium is essentially unique.

The Price of Anarchy (PoA) is the ratio between the worst Nash Equilibrium and the centralized optimal solution. On the other hand, the Price of Stability $(\mathrm{PoS})$ is the ratio between the best NE and the centralized optimal solution. Since we proved uniqueness of Nash Equilibrium, in our proposed network selection game $P_{o} A=P o S$. Hereafter we show that a lower bound for the PoA is $\frac{4}{3}$.

Proposition 3. Lower bound on the PoA. The Price of Anarchy in our proposed network selection game is $\operatorname{Po} A \geq \frac{4}{3}$.

Proof. Consider a network scenario with 2 access points and 2 mobile clients, and assume that the first AP has a rate of $r_{1}=1$, while the second has a rate of $r_{2}=2+\epsilon$, for a small $\epsilon>0$. 
The centralized optimal solution is obtained when each MC connects to one of the available APs and, in this scenario, the overall congestion is Cong ${ }_{O P T}=1+\frac{1}{2+\epsilon}$.

On the other hand, the Nash Equilibrium is obtained when both the MCs connect to the AP with the higher rate. As a matter of fact, in this configuration, each MC experiences a congestion equal to $\frac{2}{2+\epsilon}<1$, where 1 is the congestion the MC would experience if it migrated to the other access point. In the NE configuration, the overall congestion is given by $\operatorname{Cong}_{N E}=2^{2} /(2+\epsilon)$.

Therefore, since the Price of Anarchy is $\operatorname{Po} A=\frac{4 /(2+\epsilon)}{1+\frac{1}{2+\epsilon}}$, a lower bound for it is $\operatorname{Po} A \geq 4 / 3$, obtained when $\epsilon \rightarrow 0^{+}$.

To provide further evidence that the previous bound is very tight, we numerically computed values for the PoA.

For each access point $j \in \mathcal{A}$, we denote with $n_{j} \in \mathbb{Z}$ the number of mobile clients connected to $j$ in the optimal solution. The number of MCs connected to $j$ in the Nash Equilibrium is given by $n_{j}+m_{j}$, where the variable $m_{j} \in \mathbb{Z}$ is used to characterize the NE allocation, and represents the difference in the number of MCs connected to AP $j$ at equilibrium. To find the PoA, regardless of the specific scenario, we need also consider the rate of the $j$-th access point $r_{j} \in \mathbb{R}$ as a decision variable, the only parameter that we need to bound is the number of access points $|\mathcal{A}|$.

We then formulate the Mixed-Integer Non-Linear Optimization model provided hereafter:

$\max \frac{\sum_{j \in \mathcal{A}} \frac{\left(n_{j}+m_{j}\right)^{2}}{r_{j}}}{\sum_{j \in \mathcal{A}} \frac{n_{j}^{2}}{r_{j}}}$

subject to:

$$
\begin{aligned}
& \sum_{j \in \mathcal{A}} \frac{n_{j}^{2}}{r_{j}} \leq \sum_{j \in \mathcal{A}} \frac{\left(n_{j}+m_{j}\right)^{2}}{r_{j}} \\
& \sum_{j \in A} m_{j}=0 \\
& \frac{\left(n_{j}+m_{j}\right)}{r_{j}} \leq \frac{\left(n_{i}+m_{i}+1\right)}{r_{i}} \\
& n_{j}+m_{j} \geq 0 \\
& n_{j} \geq 1 \\
& r_{j} \geq 1 \\
& n_{j}, m_{j} \in \mathbb{Z} \\
& r_{j} \in \mathbb{R}
\end{aligned}
$$

The objective function (31) computes the Price of Anarchy. Constraint (32) enforces the optimality condition: the overall congestion in the optimal allocation $\sum_{j \in \mathcal{A}} \frac{n_{j}^{2}}{r_{j}}$ should be smaller or at most equal to the one experienced at equilib$\operatorname{rium} \sum_{j \in \mathcal{A}} \frac{\left(n_{j}+m_{j}\right)^{2}}{r_{j}}$. Constraint (33) makes sure that the overall number of mobile clients does not change while evaluating the Nash Equilibrium.

The Nash Equilibrium conditions are imposed in (34). In fact, by definition of the Nash Equilibrium, the congestion $\frac{\left(n_{j}+m_{j}\right)}{r_{j}}$ experienced at equilibrium by the mobile clients connected to the access point $j \in \mathcal{A}$, should be lower than the congestion they would experience if they migrated to another access point $i \neq j$, which is given by $\frac{\left(n_{i}+m_{i}+1\right)}{r_{i}}$.

In (35) we make sure that at equilibrium the number of mobile clients connected to each access point is non-negative. We assign at least one mobile client to each AP in the optimal solution, as enforced in (36). In (37) we set the minimum rate to be equal to 1; finally, integrality constraints are set in (38), whereas the rate is going to be continuous, as in (39).

We used the Bonmin solver [14] to numerically find solutions of this optimization problem. We numerically confirm that the Price of Anarchy is $P o A=4 / 3$ (i.e., the bound we provided for the PoA is tight), for each number of access points up to 50 .

\section{NUMERICAL RESUlTS}

In this section, we analyze and discuss the numerical results obtained solving our proposed models and heuristics in realistic, large-size network scenarios. More specifically, we first illustrate (Sec. VI-A) the experimental methodology used to evaluate the proposed algorithms, and then (Sec. VI-B) we discuss the obtained results.

\section{A. Methodology}

Unless stated otherwise, simulations are conducted by uniformly distributing 60 access points (APs) in a $300 \times 300 \mathrm{~m}^{2}$ grid, while the locations of 100 mobile clients (MCs) are selected as a bi-variate Gaussian distribution centered on uniformly chosen APs. The bids of the APs are selected uniformly in the $[7,15]$ USD range, whereas the demands of every $\mathrm{MC}$ are generated in the range $[0.5,3] \mathrm{Mbit} / \mathrm{s}$. We set the traffic-proportional cache miss cost $C$ to 5 USD per Mbit/s, and we consider different profit values $P$, in the set $\{5,10,15,20,25\}$ USD per Mbit/s. We performed 20 runs for every scenario, computing the narrow $95 \%$ confidence intervals reported in each figure.

We assume that the radio access network is composed of 802.11a wireless access points. Due to the path attenuation, the received power is a function of the distance $l_{i, j}$ between the mobile client (MC) $i \in \mathcal{M}$ and an access point (AP) $j \in \mathcal{A}$, and is given by:

$$
P_{r}=P_{t} g_{t} g_{r}\left(\frac{\lambda}{4 \pi}\right)^{2} l_{i, j}^{-\eta}=P_{t} g_{t} g_{r}\left(\frac{c}{4 \pi f}\right)^{2} l_{i, j}^{-\eta},
$$

where $P_{t}$ is the transmission power, $g_{t}$ and $g_{r}$ are the transmission and reception gains, respectively, $f$ is the frequency, $c$ is the speed of light and $\eta$ is the path loss exponent. The path loss is hence given by:

$$
\begin{aligned}
P L_{d B} & =10 \log _{10}\left(\frac{P_{r}}{P_{t}}\right) \\
& =10\left(\log _{10}\left(g_{t} g_{r}\right)+2 \log _{10}\left(\frac{c}{4 \pi}\right)-\log _{10}\left(f^{2} l_{i, j}^{\eta}\right)\right) .
\end{aligned}
$$

We assume that APs are equipped with the Atheros AR5413 chipset, and we will refer to the corresponding data sheet [15], to obtain the parameters characterizing the radio specification. In addition to that, we assume that gains are both unitary, 
the frequency is $5.25 \mathrm{GHz}$ and the path loss exponent is 3 . Given (41), we can compute the transmission rate by comparing the path loss value to the receiver sensitivity provided in Table III.

The Zipf discrete distribution is used to characterize the object popularity, since it was shown that it is an adequate model for it [16], [17], [18]. In particular, the Zipf model partitions the objects in $N$ groups according to their popularity rank. Let $\alpha$ be the popularity exponent of the distribution, the Zipf probability mass function (PMF) and cumulative density function (CDF) for the $n$-th popularity rank are defined as:

$$
\begin{gathered}
P(X=n)=\frac{1 / n^{\alpha}}{\sum_{i=1}^{N}\left(1 / i^{\alpha}\right)}, \\
F(n)=\sum_{i=1}^{n} P(n)=\frac{\sum_{i=1}^{n}\left(1 / i^{\alpha}\right)}{\sum_{i=1}^{N}\left(1 / i^{\alpha}\right)} .
\end{gathered}
$$

As frequently done in the literature (e.g: [19], [20], [21]) we work under the assumptions of the "Independent Reference Model” (IRM), according to which successive content requests are independent identically distributed (i.i.d.), where the popularity class of an object is a discrete random variable $X$, with probability mass function $P(X)$. We assume that cache replacement is performed according to the "Least frequently used" (LFU) policy, since under the IRM assumption, LFU is known to maximize the hit rate [19].

Let $C_{j}$ be the rank of the least popular cached objects at the $j$-th AP. Since popularity classes are sorted in decreasing order, $C_{j}$ can be computed as follows:

$$
C_{j}=\left\lfloor\frac{\text { Cache size at AP } j}{\text { Average object size }}\right\rfloor .
$$

Let $q(n)$ be the request rate of the objects belonging to the $n$-th popularity class. We can then define the hit rate $h_{j}$ for AP $j$ as:

$$
h_{j}=\frac{\sum_{1 \leq n \leq C_{j}} q(n)}{\sum_{1 \leq n \leq N} q(n)},
$$

which is the ratio between the requests for objects belonging to the $C_{j}$ most popular classes that have been stored in the caches and the requests for all the available objects.

Finally, we define the normalized cache size, $N$, as the ratio between the average cache size and the size of all the objects:

$$
N=\frac{\text { Avg. cache size }}{\text { Objects cardinality } \cdot \text { Average object size }} \text {. }
$$

For each AP, we chose the backhaul Internet bandwidth uniformly in the set $\{1 ; 6 ; 8 ; 20 ; 100\} \mathrm{Mbit} / \mathrm{s}$, while the size of the leased caching storage was uniformly selected in the range $[10,100]$ Gbytes. We consider objects having an average size of 100 Mbytes, while we take into account two catalog cardinalities equal to $10^{4}$ and $10^{6}$ objects, in line with what Fricker et al. have identified for the User Generated Content (UGC) scenario in [19]. However, for similar values of $N$,

Table III

RECEIVER SENSITIVITY OF THE ATHEROS AR5413 CHIPSET

\begin{tabular}{|l|c|c|c|c|c|c|c|c|}
\hline $\begin{array}{l}\text { Path Loss } \\
(\mathrm{dB})\end{array}$ & -72 & -73 & -77 & -81 & -84 & -86 & -88 & -90 \\
\hline $\begin{array}{l}\text { Rate } \\
(\mathrm{Mbit} / \mathrm{s})\end{array}$ & 54 & 48 & 36 & 24 & 18 & 12 & 9 & 6 \\
\hline
\end{tabular}

similar trends can be observed either by changing the object catalog size, or by increasing the amount of caching storage that is leased.

In the following experiments we set the Zipf $\alpha$ exponent to 0.9 , in line with [19]. Even though better results can be observed with higher $\alpha$ values, due to the higher skewness of the request distribution, we cautiously consider this more adverse case. Since we assume that the object cardinality can either be $10^{4}$ or $10^{6}$, the normalized cache sizes $N$ are thus equal to 0.0512 and 0.0005 respectively, meaning that caches can store up to $5.12 \%$ of the entire catalog, when $10^{4}$ objects are considered, while only $0.05 \%$ of the objects can be cached, while considering a larger catalog of $10^{6}$ objects. Results for $N=0.0051$ were also generated, but for the sake of brevity will be omitted here since they are in between the two extremes. Regarding the three variants of the algorithms presented in Table II, we denote the greedy $M C$, cache and backhaul with G.m., G.c. and G.b., respectively.

\section{B. Performance Analysis}

Unless otherwise specified, the figures illustrated in this section are related to a network scenario with 60 mobile clients (MCs) and 60 access points (APs), with a profit $P=10 \mathrm{USD}$ per Mbit/s.

Social Welfare. The social welfare metric $(S W)$ is the objective function of the ILP model (4), and has a monetary currency (USD) as unit of measurement. The value of such metric is portrayed in Fig. 3. In particular, Fig. 3a and $3 \mathrm{~b}$ show the $S W$ trend as a function of the number of APs, for a content catalog of $10^{6}(N=0.0005)$ and $10^{4}$ objects $(N=0.0512)$, respectively. Fig. $3 \mathrm{c}$ and $3 \mathrm{~d}$ consider the number of MCs, while in Fig. 3e and $3 f$ we show the SW as a function of the CP's profit per unit of bandwidth. The $S W$ has an increasing trend with respect to the number of access points $|\mathcal{A}|$, the number of mobile clients $|\mathcal{M}|$, and the average profit of the content provider $P$; in fact, when the number of APs, MCs, or the profit increase, the allocation algorithm chooses to accommodate more traffic demands since it improves the value of the $S W$ metric. We also note that, while the $S W$ has a linear trend in the number of MCs (as in Fig. 3c-3d), or the profit (as in Fig. 3e-3f), it exhibits instead a logarithmic trend in the number of APs (as in Fig. 3a-3b). This behavior is caused by the fact that the number of MCs is fixed to 60 , thus having more than 10 APs only marginally improves the $S W$ metric. On the other hand, having $|\mathcal{A}|<10$ significantly penalizes the performance of the system because the network infrastructure does not have enough capacity to serve all the clients' demands.

Another interesting observation that can be deduced by comparing Fig. $3 \mathrm{a}$ and $3 \mathrm{~b}$ is that having a larger normalized cache size of $N=0.0521$ ( $10^{4}$ objects) can lead to significant improvements on the $S W$ which is $27 \%$ higher than for $N=0.0005$ ( $10^{6}$ objects $)$, when considering 60 APs. Similar gains can be observed comparing Fig. 3c with Fig. 3d, whereas considering Fig. $3 \mathrm{e}$ and $3 \mathrm{f}$, we can conclude that the higher the profit per unit of bandwidth, the lower the gain of having a larger $N$. In particular, in this latter case, when the profit is 

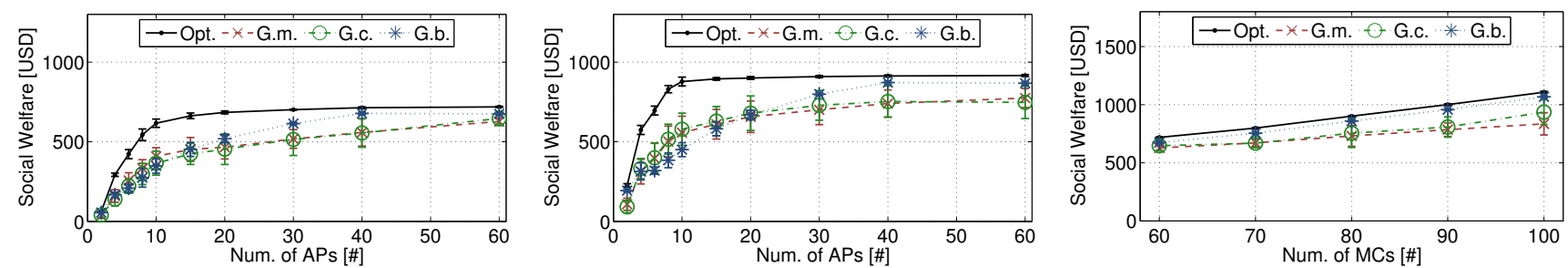

(a) $N=0.0005,|\mathcal{M}|=60, P=10 U S D$ Fun. of(b) $N=0.0512,|\mathcal{M}|=60, P=10$ USD Fun.(c) $N=0.0005,|\mathcal{A}|=60, P=10$ USD Fun. of $|\mathcal{A}|$
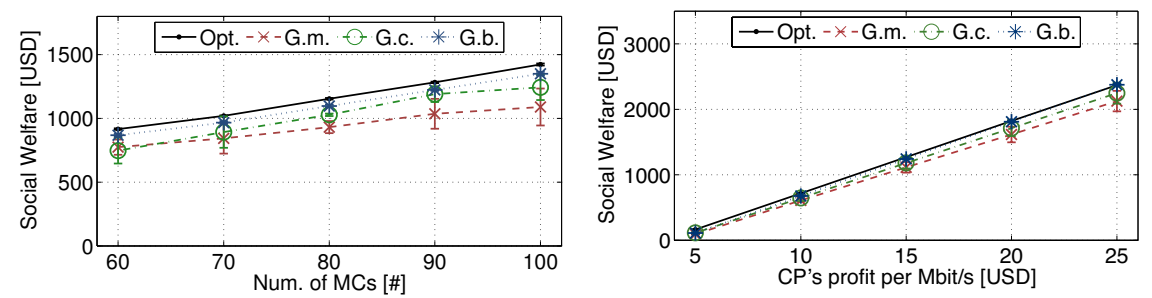

$|\mathcal{M}|$

(d) $N=0.0512,|\mathcal{A}|=60, P=10$ USD Fun. of(e) $N=0.0005,|\mathcal{M}|=60,|\mathcal{A}|=60$ Fun. of $P(\mathrm{f}) \quad N=0.0512,|\mathcal{M}|=60,|\mathcal{A}|=60$ Fun. of $P$ $|\mathcal{M}|$

Figure 3. Social Welfare, The plots show the value obtained for the Social Welfare $(S W)$ metric, comparing the value obtained with the optimal allocation algorithm and the three greedy heuristics. In particular, in Fig. $3 \mathrm{a}$ and $3 \mathrm{~b}$ we show the trend of the $S W$ as a function of the number of APs, for a catalog of $10^{6}(N=0.0005)$ and $10^{4}(N=0.0512)$ objects, respectively. Fig. $3 \mathrm{c}$ and $3 \mathrm{~d}$ consider the number of MCs, whereas Fig. $3 \mathrm{e}$ and $3 \mathrm{f}$ are functions of the profit.

25USD per Mbit/s, the gain for $N=0.0512$ is only $9 \%$ with respect to $N=0.0005$.

Finally, comparing the values obtained for the optimal allocation algorithm and our proposed heuristics, we observe in Fig. 3e-3f that, when considering the $S W$ as a function of the profit, the heuristics compute a close-to-optimal value for the objective function, losing, in the worst case, only $16 \%$ of $S W$. In particular, the greedy backhaul (G.b.) allocation algorithm seems to perform slightly better than the other two, efficiently computing a solution on average less than $10 \%$ lower than the optimum. Similar conclusions regarding the greedy heuristics can be derived from Fig. 3a-3f.

Leasing Cost. The leasing cost metric $(L C)$ is defined as the total cost sustained by the content provider (CP) to lease the subset of access points selected by the mechanism, and is equal to $L C=\sum_{j \in \mathcal{A}} y_{j} p_{j}$. The trend of $L C$ as a function of the number of access points is shown in Fig. 4a for a normalized cache size of $N=0.0005$ ( $10^{6}$ objects $)$ and in Fig. 4b for $N=0.0512\left(10^{4}\right.$ objects $)$. It is important to stress the fact that the price the content provider pays to the access point (AP) owners is not the amount that was bid, but it is computed in a way as to make sure that both the optimal algorithm and the heuristics enforce the individual rationality and truthfulness properties.

By comparing Fig. $4 a$ and $4 b$, we observe that the normalized cache size $N$ only partially affects the $L C$ metric. Moreover, when the number of APs is large, the optimal allocation algorithm outperforms the heuristics, paying the AP owners $52 \%$ less than the greedy cache (G.c.) solution, and up to $95 \%$ less compared to the greedy backhaul (G.b.) with $N=0.0521$.

As we will discuss in the following for the Unserved

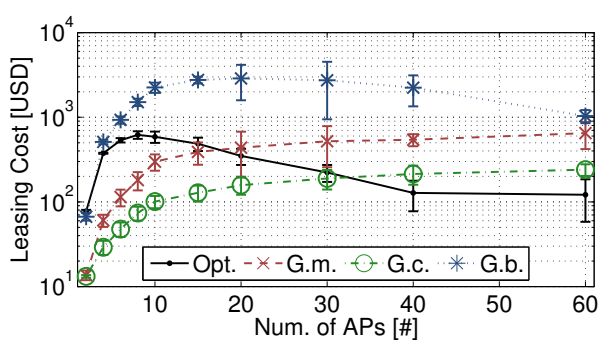

(a) $N=0.0005,|\mathcal{M}|=60, P=10$ USD $-L C$

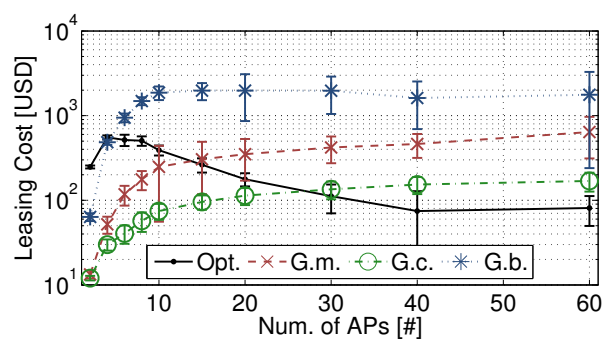

(b) $N=0.0512,|\mathcal{M}|=60, P=10$ USD $-L C$

Figure 4. Leasing Cost, The figures show the Leasing Cost $(L C)$, as a function of the number of APs. In Fig. 4a the normalized cache size was set to $N=$ 0.0005 , whereas in Fig. 4b it was set to $N=0.0521$.

Requests $(U R)$ metric, when the number of APs is smaller than 10, not all the MC's requests can be served by the available network capacity, and the local maximum that is observed for the optimal allocation algorithm when the number of APs is around 9 is caused exactly by this reason. As a matter of fact, in this scenario, by removing one of the few APs deployed, the overall traffic request that the network can serve will be significantly reduced, making the $S W$ decrease sharply. In other words, the role of every single AP that is 


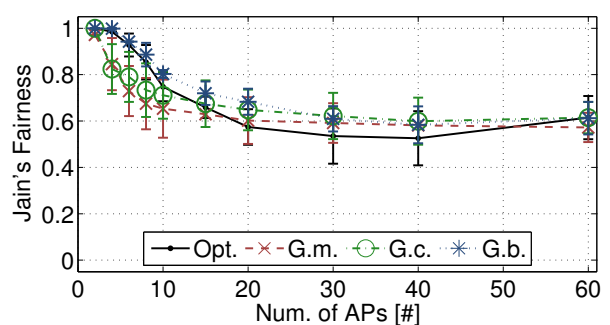

(a) $N=0.0005,|\mathcal{M}|=60, P=10$ USD $-f$

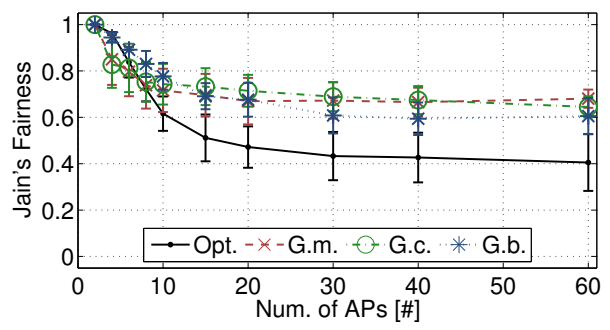

(b) $N=0.0512,|\mathcal{M}|=60, P=10 \mathrm{USD}-f$

Figure 5. Jain's Fairness Index, Fig. 5a shows Jain's Fairness Index $f$ for $N=0.0005$, as a function of the number of APs, whereas in Fig. $5 \mathrm{~b}$ we portrait the result for $N=0.0512$.

selected by the allocation mechanism is crucial, and therefore the price the allocation procedure pays is much larger. On the other hand, when the number of APs increases, the role of every single AP is much less critical and the payment they receive is accordingly much lower.

Jain's Fairness Index. The Jain's Fairness Index $(f)$ is defined according to [22] as:

$$
f=\left(\sum_{\forall j \in \mathcal{A}} \rho_{j}\right)^{2} /\left(\sum_{\forall j \in \mathcal{A}} y_{j}\right) \cdot \sum_{\forall \in \mathcal{A}} \rho_{j}^{2}
$$

where $\rho_{j}=\frac{y_{j} p_{j}}{\sum_{i \in \mathcal{M}} x_{i, j} d_{i}}$ is the ratio between the price paid to lease AP $j$ and the overall demand it is serving. The performance of the optimal and greedy allocation algorithms with respect to the $f$ metric is depicted in Fig. 5, where in 5a we set the normalized cache size $N=0.0005$ ( $10^{6}$ objects), whereas in $5 \mathrm{~b}, N=0.0512$ ( $10^{4}$ objects).

By comparing the two figures, we can state that the greedy heuristics have a similar trend with respect to the fairness metric, which is rather insensitive to the normalized cache size. As a matter of fact, in both Fig. 5a and $5 \mathrm{~b}$ we observe that the heuristics generate a solution that is on average $62 \%$ fair, when the number of $\mathrm{AP}$ is $|\mathcal{A}|=60$. At the same time, the optimal allocation algorithm leads to worse solutions in terms of the fairness metric, especially when having a larger cache. In fact, in this latter case, as shown in Fig. 5b, the fairness of the optimal allocation algorithm stabilizes at $40 \%$ when 60 APs are considered.

Completion Time. The effect of the completion time $(t)$ is shown in Fig. 6. As usual, Fig. 6a refers to $N=0.0005\left(10^{6}\right.$ objects) while Fig. $6 \mathrm{~b}$ is for $N=0.0512\left(10^{4}\right.$ objects). First of all, by comparing Fig. $6 \mathrm{a}$ with $6 \mathrm{~b}$, we can conclude that the size of the catalog, and therefore the average AP's hit-rate has a negligible impact on the observed completion time. On top of that, the optimal allocation algorithm requires much more time than the one spent using our proposed heuristics, which

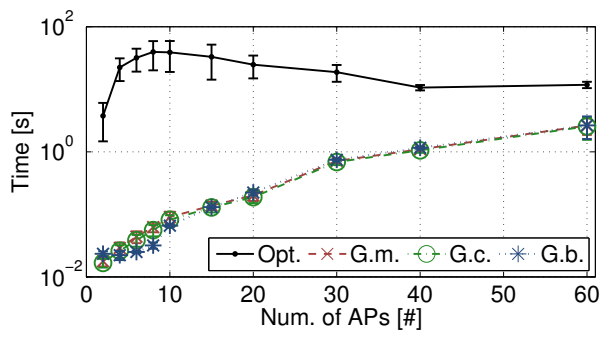

(a) $N=0.0005,|\mathcal{M}|=60, P=10 \mathrm{USD}-t$

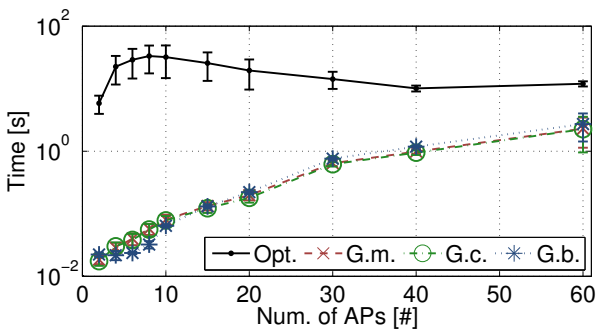

(b) $N=0.0512,|\mathcal{M}|=60, P=10$ USD - $t$

Figure 6. Completion Time, The completion time $t$ metric is shown as a function of the number of APs. In Fig. 6a we report the results for $N=$ 0.0005 , whereas Fig. $6 \mathrm{~b}$ refers to $N=0.0512$.

are all characterized by practically the same computing time.

The time trend of the optimal allocation algorithm not only has a local maximum when the number of access points $|\mathcal{A}|$ is equal to 9 , but it also exhibits large confidence intervals when $|\mathcal{A}|<10$. The reason for both these behaviors lies in the fact that when there are few access points (APs), the allocation will not serve all the clients' demands, since the spare network capacity is not sufficient to do so. Moreover, the choice of which AP should be used becomes critical, and more time is required by the algorithm in order to explore the combinatorial state space of the solutions.

Saved Bandwidth, Unserved Requests, Hit Rate. Hereafter we jointly discuss the results we obtained for the $S B$, $U R$ and $\bar{h}$ metrics. In particular, the Bandwidth Saved for Caching $(S B)$ is defined as the total amount of traffic directly served by the caches, measured in Mbit/s and equal to $S B=\sum_{\substack{\forall i \in \mathcal{M} \\ \forall j \in \mathcal{A}}} x_{i, j} d_{i} h_{j}$. The $U R$ metric is instead the Fraction of Unserved Requests, which is the fraction of the mobile clients' traffic demands that are not accommodated according to the allocation algorithm, and is equal to $U R=\frac{\sum_{\forall i \in \mathcal{M}}\left(1-\max _{\forall j \in \mathcal{A}} x_{i, j}\right) d_{i}}{\sum_{\forall i \in \mathcal{M}} d_{i}}$. Finally, the Average Hit Rate $\bar{h}$ is defined as the ratio between the content served by the caches and the sum of all the served clients' demands, and is

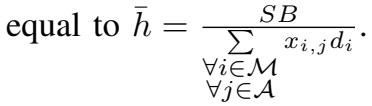

The Saved Bandwidth $(S B)$ trend, shown in Fig. 7a and $7 \mathrm{~b}$ is logarithmic in the number of APs deployed. However, the average hit rate $\bar{h}$ observed is constant in the number of access points and equal to $41 \%$ with a normalized cache size of $N=0.0005$ and $78 \%$ when $N=0.0521$, for both the optimal allocation algorithm as well as the heuristics. The 


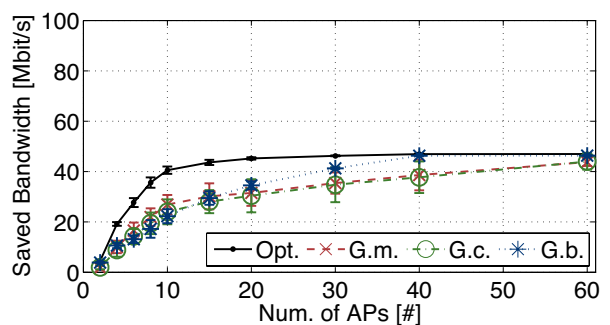

(a) $N=0.0005,|\mathcal{M}|=60, P=10 \mathrm{USD}-S B$

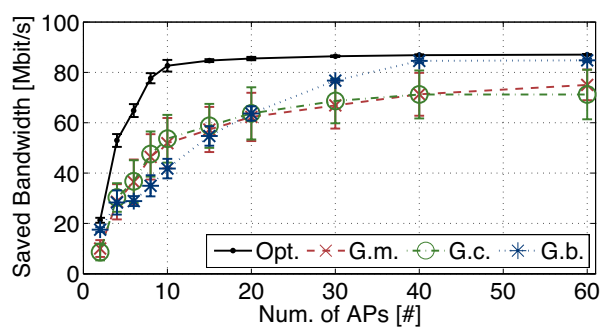

(b) $N=0.0512,|\mathcal{M}|=60, P=10$ USD $-S B$

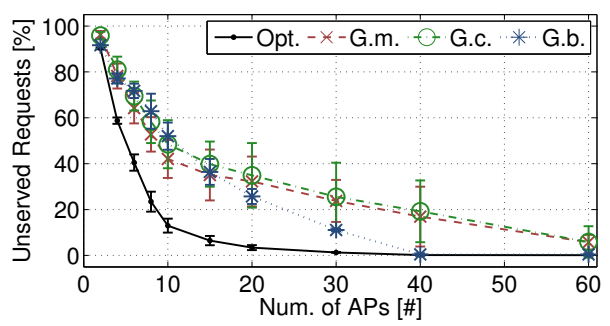

(c) $N=0.0005,|\mathcal{M}|=60, P=10 \mathrm{USD}-U R$

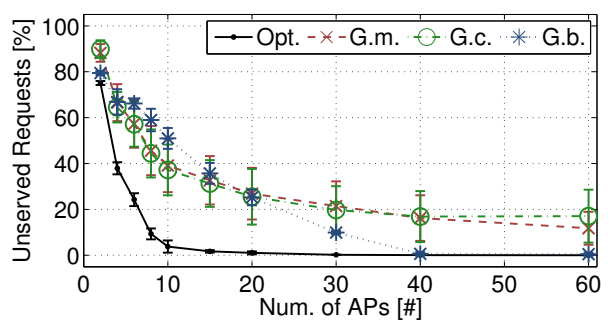

(d) $N=0.0512,|\mathcal{M}|=60, P=10 \mathrm{USD}-U R$

Figure 7. Saved Bandwidth, Unserved Requests, Fig. 7a and 7b show the Saved Bandwidth metric as a function of the number of APs, for $N=0.0005$ and $N=0.0521$, respectively. The trend of the fraction of Unserved Requests is instead depicted in Fig. $7 \mathrm{c}$ and $7 \mathrm{~d}$.

constant value for $\bar{h}$ and the logarithmic trend for $S B$ are caused by the fact that our proposed formulation admits partial coverage, that is, not all the requests of the mobile clients (MCs) are served by the network. In particular, as Fig. 7c and 7 d show, when $N=0.0005,10$ APs can serve up to $84 \%$ of the overall demand (using the optimal algorithm), while $96 \%$ is reached when $N=0.0521$. Since the bottleneck in our system model is the backhaul internet connection of every access point, deploying some caching storage in the system makes the network accommodate more traffic. On top of that, 40 APs are necessary to make all the client's requests be served with $N=0.0005$ (10 $0^{6}$ objects), whereas 30 APs are sufficient if we consider $N=0.0521$ ( $10^{4}$ objects). In terms of the unserved requests, the greedy backhaul very well approaches

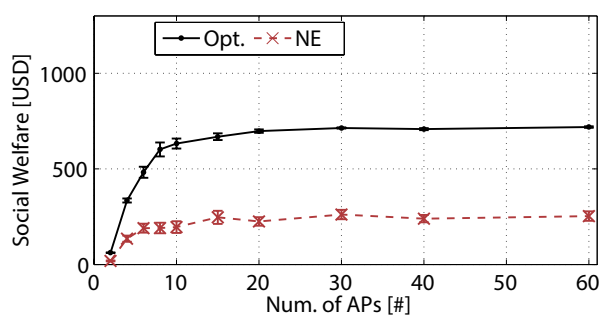

(a) $N=0.0005,|\mathcal{M}|=60, P=10$ USD Fun. of $|\mathcal{A}|$

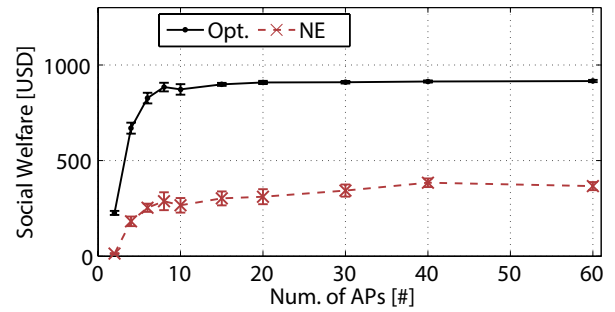

(b) $N=0.0521,|\mathcal{M}|=60, P=10$ USD Fun. of $|\mathcal{A}|$

Figure 8. Social Welfare - Network Selection Game, The figures show a comparison of the Social Welfare $(S W)$ obtained in the centralized (Opt.) and the distributed scenario (Nash Equilibrium, $N E$ ), in particular they show the performance loss due to the distributed allocation of the mobile clients. Fig. 8a represents the scenario with an object catalog of $10^{6}(N=0.0005)$, while Fig. $8 \mathrm{~b}$ refers to the case where the object catalog contains $10^{4}$ objects $(N=0.0521)$, varying the number of APs.

the results observed for the optimal solution, whereas the other two heuristic algorithms serve on average $18 \%$ less traffic demands, when $N=0.0521$ and $|\mathcal{A}|=60$.

As we have already commented for the $S W, L C$ and $t$ metric, the threshold of 10 APs clearly marks two different operating zones: when a large amount of traffic requests cannot be served by the networking infrastructure, this has a major impact on the multiple metrics characterizing the overall system performance.

Social Welfare - Network Selection Game. Fig. 3 reports the $S W$ when the allocation is performed in the centralized setting, both optimally and using our proposed heuristic. Instead, in Fig. 8, we consider the distributed case and we show the $S W$ when MCs reach the unique Nash Equilibrium of the network selection game we presented in Sec. V. By comparing the value of the centralized optimal $S W$ in Fig. 8a and $8 \mathrm{~b}$, we can state that in the distributed scenario we lose ${ }^{1}$ up to $65 \% S W$ when $N=0.0005$ ( $10^{6}$ objects $)$, and $60 \%$ when $N=0.0512\left(10^{4}\right.$ objects $)$, compared to the optimum (obtained with the ILP model (4)-(10)).

For the sake of brevity, we omit the results regarding the convergence speed of the best response dynamics for the network selection game. Indeed, in all the considered scenarios the game always reaches the equilibrium in less than 10 iterations.

\section{RELATED WORK}

In this section, we survey related works. In particular Sec. VII-A reviews the ICN design principles; Sec. VII-B

\footnotetext{
${ }^{1}$ Please note that this evaluation is done with respect to the social welfare SW, whereas in Sec. V we computed the Price of Anarchy with respect to the network congestion only.
} 
presents relevant incentive mechanisms for communication networks, while Sec. VII-C surveys proposals for mobile data offloading through WiFi.

\section{A. Information Centric Network Solutions}

Information Centric Networks have been designed with the ambitious goal to provide a new networking paradigm that could better accommodate new performance, security, mobility and scalability requirements imposed on the Internet by its users. Several architectures for ICNs have been proposed in the literature and in ongoing research projects: DONA [23], CONET [24], PURSUIT [25], and NDN/CCN [26].

For the sake of simplicity, we specifically design our proposal for NDN/CCN (Named Data Networking, ContentCentric Networking), since a widely used and open implementation of this model is available [27]. Additionally, to the best of our knowledge, it is the proposal that has received most of the attention from the scientific community. However, we would like to point out the fact that our model can also be adapted to other ICN designs in a straightforward way, since the only requirement that needs to be enforced is the possibility to implement content caching on an access point.

Addressing the content rather than the location has the main advantage of improving the performance by making universal in-network caching, as well as multicast delivery, easily implementable in the network architecture [28]. On top of that, also clients mobility will enjoy positive benefits, as described by Tyson et al. in [29]. For this reason, it becomes vital to understand the effect of in-network caching in order to study the behavior of an ICN. In particular, in [19], Fricker et al. propose a model to compute the hit rate for a single and two-layer cache hierarchy, given a Zipf content popularity distribution. In [30], Psaras et al. studied the caching performance for a tree-like $\mathrm{CCN}$ topology, by means of representing the state of every router using Markov chains. Rosensweig et al. present in [31] a multi-cache approximation algorithm for a network of caches using the LRU replacement policy, that can be used to study the performance of generaltopology cache networks.

Rather than merely studying the cache hit rate, in this work we consider the economic benefits that the content provider can experience. In particular, with our proposal the content provider will be able to jointly increase the number of mobile clients reached by his service, while opportunistically exploiting content caching to reduce the load on his infrastructure.

\section{B. Incentive Mechanisms in Communication Networks}

Incentive mechanisms and auction theory have been used to design efficient allocation mechanisms in several network contexts. In particular, as described in [32], they are extremely appealing to model, for example, the problem of leasing underutilized spectrum to secondary users, tackled in cognitive radio networks (e.g: [33], [34], [35]).

Vickrey auctions are used in [32] to make the cognitive radio users compete to acquire the radio resources; incentives are paid to the primary users for sharing their licensed spectrum. In [36] Jia et al. formulate a revenue maximization auction mechanism for a primary license holder, using the VickreyClarke-Groves (VCG) mechanism. In [37] the authors propose to use a knapsack-based auction model to foster the dynamic spectrum allocation to secondary wireless service providers to maximize revenue and spectrum usage. Dynamic spectrum leasing is also the focus of [38], where secondary service providers lease spectrum from brokers to provide network connectivity to secondary users. Besides these classical scenarios, auctions are becoming an even more interesting tool to model bandwidth allocation. Dai et al. in [39] present a collaborative caching auction system for wireless video streaming based on the VCG mechanism. Their proposal fosters the cooperation between cache servers by ensuring that every bidder declares his real private valuation for the auctioned resource, thus ensuring the truthfulness property.

In [40] Liu et al. propose a distributed power-allocation algorithm for wireless networks to let users collaboratively choose the transmission power they use. The work is presented as an auction mechanism, and the authors compute the optimal allocation strategy, although nor individual rationality nor truthfulness are analyzed or discussed. Finding a truthful mechanism with approximate social-welfare or revenue is instead the focus of [41], where the authors consider the dynamic spectrum access for both spatial and temporal dimensions. In a similar context, Wu et al. design in [42] an auction mechanism that ensures Pareto-optimal relay power allocation, competitive fairness among users, and convergence on the distributed implementation. Lastly, Mukherjee presents in [43] a work where a truthful, Pareto-optimal auction mechanism is proposed to handle energy management from a battery shared among multiple users.

Meanwhile, the migration to the novel paradigm of ICN will greatly modify the nature of economic interactions between different parties at the network-wide scale. In particular, Rajahalme et al. have thoroughly described in [44] the changes that will likely incur at the inter-domain point of view, when updating the infrastructure from a TCP/IP network to ICN. Pham et al. have modeled in [45] the economic interactions between the different actors of an Information Centric Network, using the framework of game theory. Game theory is also used in [46] to propose an incentive scheme to foster cache cooperation and users' collaboration for multimedia streaming purposes. In particular, the authors tackle the problem as a resource allocation game in both the cooperative as well as the noncooperative settings.

Unlike the surveyed literature, our approach is to tackle the mechanism design problem, while jointly taking into account the available bandwidth as well as the storage space offered by every access point owner. Given the fact that we study the performance of this mechanism for an ICN, the availability of caches not only reduces the backhaul capacity used at every access point, but it is also beneficial to the content provider himself, since it offloads its infrastructure.

\section{Mobile Data Offloading Through WiFi}

Mobile data offloading through WiFi networks is frequently envisioned in the literature as an efficient technique to serve 
the ever increasing bandwidth requirements expressed by endusers with mobile devices. In particular, Lee et al. show in [47] that nowadays, WiFi connections offload up to $65 \%$ of the total mobile data traffic, mostly thanks to the broadband connectivity provided by residential access points.

The scenario in which access point owners offer their unexploited wireless connectivity to other users has attracted the attention of several companies such as FON [48], Wisher [49] and also Skype WiFi [50], [51]. While Skype WiFi is mostly targeting hotspots in public places such as hotels, restaurants, train stations and airports, FON and Wisher also consider residential users. Access point owners that choose to provide wireless connectivity usually gain the privilege of using the wireless connection provided by other users in the community, but sometimes they will be also remunerated for sharing their physical resources.

Security must be guaranteed for the practical application of WiFi sharing. Leroy et al. design in [52] SWISH, a mechanism used to guarantee the safe and secure operation of WiFi sharing. SWISH not only ensures anonymity of the visiting users, but can also enforce accounting for commercial purposes, while letting regulatory organizations check what type of actions roaming users perform when they are connected to access points owned by other users.

By applying on a global scale the WiFi sharing model, one can also envision the realization of Public Access WiFi Networks (PAWS). A feasibility study for PAWS is presented in [53]. The key findings discovered by the authors show that many domestic broadband connections have enough spare capacity to share the available WiFi connectivity; furthermore they also found out that one such type of service is going to be exploited in many different ways by users, and its adoption will likely be very relevant. Similar conclusions were also envisioned by Landa-Torres et al. in [54], where the authors focus instead on the network planning problem, to maximize the overall signal coverage, under a budgetconstrained condition.

Lastly, the problem of incentive mechanisms to remunerate AP owners is presented in [55], [56]. The focus of [55] is to consider the scenario in which mobile user equipments behave as wireless access point and provide network connectivity through $3 \mathrm{G} / 4 \mathrm{G}$ connections. While in [56], the authors formulate a double auction for a fixed network.

The surveyed literature shows that there is a clear need to consider the scenario where many access point owners lease their unexploited physical resources through WiFi. However, the novelty of our contribution is to consider a system model that focuses on the content distribution problem. In particular we consider the case in which AP owners jointly offer network connectivity as well as caching storage. We strongly believe that adequate incentives should be provided to AP owners in exchange of the resources they lease, moreover the presence of the distributed caches will benefit both roaming users, as well as the AP owners, by reducing the congestion on the backhaul Internet connection, and improving the QoE. Our belief is supported by the effort that equipment manufacturers and mobile operators are investing on the analysis of caching at different layers of the network infrastructure as a means to meet the tight constraints of 5G networks [57], [58].

\section{CONCLUSION}

This paper proposed a novel mechanism for Information Centric Networks to stimulate wireless access point owners to jointly lease their unused bandwidth and storage space to a content provider, under the partial coverage constraints. By jointly leasing the spare bandwidth and caching storage offered by access points owners, the content provider can increase the number of mobile clients reached by his service, while offloading his distribution infrastructure by replicating the provided contents closer to the users' location.

We provided an algorithm to determine the optimal allocation of mobile clients to access points that ensures the individual rationality as well as the truthfulness properties by forcing the AP owners to bid the real valuation for the offered resources. We showed that the optimal allocation problem is NP-hard, and provided three efficient alternatives of a greedy algorithm that compute a sub-optimal solution of the problem in polynomial time, while still guaranteeing the individual rationality as well as the truthfulness properties.

We extended our contribution by comparing the result of the centralized allocation to the distributed case where the mobile clients are free to autonomously select the access point they prefer to use, taking into account real channel properties and the MAC behavior of the 802.11 technology. Specifically, we modeled the interactions between different users as a non-cooperative network selection game, considering as utility function the achievable throughput in loaded conditions. Furthermore, we proved that the game exhibits desirable properties: 1) existence and convergence to the pure Nash equilibrium, since it is weighted potential; 2) uniqueness of one such Nash equilibrium and, 3) we also provide a lower bound on the price of anarchy.

Finally, numerical results demonstrated that the performance of the greedy algorithms well approaches the results obtained by the optimal solution. In particular, in the worst case we considered, the heuristics computed a solution whose social welfare was only $16 \%$ lower than the optimal counterpart, while being several order of magnitudes faster that the optimal allocation algorithm.

\section{REFERENCES}

[1] M. Gritter and D. R. Cheriton, "An Architecture for Content Routing Support in the Internet," Proc. of the 3rd USENIX Symposium on Internet Technologies and Systems (USITS), San Francisco, CA, USA, Mar. 2001.

[2] J. Pan, S. Paul, and R. Jain, "A Survey of the Research on Future Internet Architectures,” IEEE Communications Magazine, vol. 49, no. 7, pp. 2636, July 2011.

[3] V. Jacobson, D. K. Smetters, J. D. Thornton, M. Plass, N. Briggs, and R. Braynard, "Networking Named Content," Communications of the $A C M$, vol. 55, no. 1, pp. 117-124, 2012.

[4] http://www.amazon.com/gp/help/customer/display.html?nodeId= 200505540, Amazon Kindle Wireless Connections, Last accessed: Dec. 2015.

[5] B. Hu, H. Zhao, and H. Jiang, "Wireless Multicast Using Relays: Incentive Mechanism and Analysis," IEEE Transactions on Vehicular Technology, vol. 62, no. 5, pp. 2204-2219, Jun 2013.

[6] J. Konka, I. Andonovic, C. Michie, and R. Atkinson, "Auction-Based Network Selection in a Market-Based Framework for Trading Wireless Communication Services," IEEE Transactions on Vehicular Technology, vol. 63, no. 3, pp. 1365-1377, March 2014. 
[7] N. Nisan, T. Roughgarden, E. Tardos, and V. V. Vazirani, Algorithmic Game Theory. Cambridge University Press, 2007.

[8] S. Martello and P. Toth, Knapsack Problems: Algorithms and Computer Implementations. New York, NY, USA: John Wiley \& Sons, Inc., 1990.

[9] D. Niyato and E. Hossain, "Dynamics of Network Selection in Heterogeneous Wireless Networks: An Evolutionary Game Approach," IEEE Transactions on Vehicular Technology, vol. 58, no. 4, pp. 2008-2017, May 2009.

[10] J. Elias, F. Martignon, A. Capone, and E. Altman, "Non-Cooperative Spectrum Access in Cognitive Radio Networks: a Game Theoretical Model," Elsevier Computer Networks, vol. 55, no. 17, pp. 3832 - 3846, 2011.

[11] M. Heusse, F. Rousseau, G. Berger-Sabbatel, and A. Duda, "Performance Anomaly of 802.11 b," in Proc. of IEEE Int.l Conf. on Computer Communications (INFOCOM), vol. 2, San Francisco, CA, USA, Mar. 2003, pp. 836-843.

[12] D. Monderer and L. S. Shapley, "Potential games," Games and economic behavior, vol. 14, no. 1, pp. 124-143, 1996

[13] M. R. Bussieck and S. Vigerske, "MINLP solver software," Wiley Encyclopedia of Operations Research and Management Science, 2010.

[14] P. Bonami, L. T. Biegler, A. R. Conn, G. Cornuéjols, I. E. Grossmann, C. D. Laird, J. Lee, A. Lodi, F. Margot, N. Sawaya et al., "An Algorithmic Framework for Convex Mixed Integer Nonlinear Programs," Discrete Optimization, vol. 5, no. 2, pp. 186-204, 2008.

[15] http://www.zcomax.com/files/AG623-49.pdf, Atheros AR5413 Data sheet Last accessed: Dec. 2015.

[16] M. Gallo, B. Kauffmann, L. Muscariello, A. Simonian, and C. Tanguy, "Performance Evaluation of the Random Replacement Policy for Networks of Caches," Performance Evaluation Journal, Elsevier, vol. 72, pp. 16-36, 2014.

[17] M. Cha, H. Kwak, P. Rodriguez, Y.-Y. Ahn, and S. Moon, "I Tube, You Tube, Everybody Tubes: Analyzing the World's Largest User Generated Content Video System," in Proc. of the 7th ACM SIGCOMM Internet Measurement Conference (IMC), San Diego, CA, USA, Oct. 2007, pp. $1-14$.

[18] S. Vanichpun and A. M. Makowski, "The Iutput of a Cache Under the Independent Reference Model: Where did the Locality of Reference go?" in ACM SIGMETRICS Performance Evaluation Review, vol. 32, no. 1. ACM, 2004, pp. 295-306.

[19] C. Fricker, P. Robert, J. Roberts, and N. Sbihi, "Impact of Traffic Mix on Caching Performance in a Content-Centric Network," Proc. of IEEE Int.l Conf. on Computer Communications (INFOCOM), NOMEN Workshop, pp. $310-315$, Orlando, FL, USA, Mar. 2012.

[20] E. J. Rosensweig, D. S. Menasche, and J. Kurose, "On the SteadyState of Cache Networks," in Proc. of IEEE Int.l Conf. on Computer Communications (INFOCOM). IEEE, Turin, Italy, Apr. 2013, pp. 863871

[21] G. Carofiglio, M. Gallo, L. Muscariello, and D. Perino, "Modeling Data Transfer in Content-Centric Networking," in Proc. of the 23rd IEEE Int.l Teletraffic Congress, San Francisco, CA, USA, Sep. 2011, pp. 111-118.

[22] R. Jain, The Art of Computer Systems Performance Analysis: Techniques for Experimental Design, Measurement, Simulation, and Modeling, ser. Wiley professional computing. Wiley, 1991.

[23] T. Koponen, M. Chawla, B.-G. Chun, A. Ermolinskiy, K. H. Kim, S. Shenker, and I. Stoica, "A Data-Oriented (and Beyond) Network Architecture," SIGCOMM Comput. Commun. Rev., vol. 37, no. 4, pp. 181-192, Aug. 2007.

[24] A. Detti, N. Blefari Melazzi, S. Salsano, and M. Pomposini, "CONET: a Content Centric Inter-Networking Architecture," Proc. of the ACM SIGCOMM Workshop on Information-centric networking, pp. 50-55, Toronto, Ontario, Canada, Aug. 2011.

[25] "FP7 PURSUIT Project," http://www.fp7-pursuit.eu, Last accessed: Dec. 2015.

[26] V. Jacobson, D. K. Smetters, N. H. Briggs, M. F. Plass, P. Stewart, J. D. Thornton, and R. L. Braynard, "VoCCN: Voice-Over Content-Centric Networks," Workshop on Re-architecting the internet, pp. 1-6, Rome, Italy, Dec. 2009.

[27] "CCNx Website," https://www.ccnx.org, Last accessed: Dec. 2015.

[28] G. Carofiglio, G. Morabito, L. Muscariello, I. Solis, and M. Varvello, "From Content Delivery Today to Information Centric Networking," Computer Networks, vol. 57, no. 16, pp. 3116-3127, 2013.

[29] G. Tyson, N. Sastry, I. Rimac, R. Cuevas, and A. Mauthe, "A Survey of Mobility in Information-Centric Networks: Challenges and Research Directions," in Proc. of the 1st ACM Workshop on Emerging Name-Oriented Mobile Networking Design-Architecture, Algorithms, and Applications, Hilton Head Island, SC, USA, Jun. 2012, pp. 1-6.
[30] I. Psaras, R. G. Clegg, R. Landa, W. K. Chai, and G. Pavlou, "Modelling and Evaluation of CCN-caching Trees," Proc. of the 10th Int.l IFIP TC 6 Conf. on Networking (NETWORKING), pp. 78-91, Valencia, Spain, May 2011.

[31] E. J. Rosensweig, J. Kurose, and D. Towsley, "Approximate Models for General Cache Networks," in Proc. of IEEE Int.l Conf. on Computer Communications (INFOCOM), San Diego, CA, USA, Mar. 2010, pp. 1-9.

[32] H.-B. Chang and K.-C. Chen, "Auction-Based Spectrum Management of Cognitive Radio Networks," IEEE Transactions on Vehicular Technology, vol. 59, no. 4, pp. 1923-1935, 2010.

[33] X. Zhou and H. Zheng, "TRUST: A General Framework for Truthful Double Spectrum Auctions," Proc. of IEEE Int.l Conf. on Computer Communications (INFOCOM), pp. 999 -1007, Rio de Janeiro, Brazil, Apr. 2009.

[34] G. S. Kasbekar and S. Sarkar, "Spectrum Auction Framework for Access Allocation in Cognitive Radio Networks," IEEE/ACM Transactions on Networking, vol. 18, no. 6, pp. 1841-1854, 2010.

[35] S. Dixit, S. Periyalwar, and H. Yanikomeroglu, "Secondary User Access in LTE Architecture Based on a Base-Station-Centric Framework With Dynamic Pricing," IEEE Transactions on Vehicular Technology, vol. 62 no. 1, pp. 284-296, Jan 2013.

[36] J. Jia, Q. Zhang, Q. Zhang, and M. Liu, "Revenue Generation for Truthful Spectrum Auction in Dynamic Spectrum Access," in Proc. of the 10th ACM Int.l Symposium on Mobile Ad Hoc Networking and Computing (MobiHoc). ACM, New Orleans, LA, USA, May 2009, pp. 3-12.

[37] S. Sengupta and M. Chatterjee, "An Economic Framework for Dynamic Spectrum Access and Service Pricing," IEEE/ACM Transactions on Networking (TON), vol. 17, no. 4, pp. 1200-1213, 2009.

[38] K. Zhu, D. Niyato, P. Wang, and Z. Han, "Dynamic Spectrum Leasing and Service Selection in Spectrum Secondary Market of Cognitive Radio Networks," IEEE Transactions on Wireless Communications, vol. 11, no. 3, pp. 1136-1145, 2012.

[39] J. Dai, F. Liu, B. Li, B. Li, and J. Liu, "Collaborative Caching in Wireless Video Streaming Through Resource Auctions," IEEE Journal on Selected Areas in Communications, pp. 458 -466, 2012.

[40] Y. Liu, M. Tao, and J. Huang, "An Auction Approach to Distributed Power Allocation for Multiuser Cooperative Networks," IEEE Transactions on Wireless Communications, vol. 12, no. 1, pp. 237-247, January 2013.

[41] M. Al-Ayyoub and H. Gupta, "Truthful Spectrum Auctions With Approximate Social-Welfare or Revenue," IEEE/ACM Transactions on Networking, vol. 22, no. 6, pp. 1873-1885, Dec 2014.

[42] D. Wu, Y. Cai, and M. Guizani, "Auction-Based Relay Power Allocation: Pareto Optimality, Fairness, and Convergence," IEEE Transactions on Communications, vol. 62, no. 7, pp. 2249-2259, July 2014.

[43] A. Mukherjee, "Energy Reallocation in a Multi-User Network With a Shared Harvesting Module and Storage Battery," IEEE Communications Letters, vol. 19, no. 2, pp. 279-282, Feb 2015.

[44] J. Rajahalme, M. Särelä, P. Nikander, and S. Tarkoma, "IncentiveCompatible Caching and Peering in Data-Oriented Networks," in Proc. of the ACM CoNEXT Conference, Madrid, Spain, Dec. 2008, p. 62.

[45] T.-M. Pham, S. Fdida, and P. Antoniadis, "Pricing in Information-Centric Network Interconnection," in Proc. of IFIP Networking Conference, Brooklyn, NY, USA, May 2013, pp. 1-9.

[46] A. T. Ip, J. Lui, and J. Liu, "A Revenue-Rewarding Scheme of Providing Incentive for Cooperative Proxy Caching for Media Streaming Systems," ACM Transactions on Multimedia Computing, Communications, and Applications (TOMCCAP), vol. 4, no. 1, p. 5, 2008.

[47] K. Lee, J. Lee, Y. Yi, I. Rhee, and S. Chong, "Mobile Data Offloading: How Much Can WiFi Deliver?" IEEE/ACM Transactions on Networking (TON), vol. 21, no. 2, pp. 536-550, 2013.

[48] "Fon Website," https://www.fon.com/, Last accessed: Dec. 2015.

[49] "Wisher," https://www.crunchbase.com/organization/whisher, Last accessed: Dec. 2015.

[50] "Skype WiFi Website," http://www.skype.com/en/features/skype-wifi/, Last accessed: Dec. 2015.

[51] L. Duan, J. Huang, and B. Shou, "Optimal Pricing for Local and Global WiFi Markets," in Proc. of IEEE Int.l Conf. on Computer Communications (INFOCOM), Turin, Italy, April 2013, pp. 1088-1096.

[52] D. Leroy, G. Detal, J. Cathalo, M. Manulis, F. Koeune, and O. Bonaventure, "SWISH: Secure WiFi Sharing," Computer Networks, vol. 55, no. 7, pp. 1614-1630, 2011.

[53] A. Sathiaseelan, R. Mortier, M. Goulden, C. Greiffenhagen, M. Radenkovic, J. Crowcroft, and D. McAuley, "A Feasibility Study of an In-the-Wild Experimental Public Access WiFi Network," in Proc. of the 5th ACM Symposium on Computing for Development, 
ser. ACM DEV-5 '14, 2014, pp. 33-42. [Online]. Available: http://doi.acm.org/10.1145/2674377.2674383

[54] I. Landa-Torres, S. Gil-Lopez, J. Del Ser, S. Salcedo-Sanz, D. Manjarres, and J. A. Portilla-Figueras, "Efficient Citywide Planning of Open WiFi Access Networks Using Novel Grouping Harmony Search Heuristics," Engineering Applications of Artificial Intelligence, vol. 26, no. 3, pp. 1124-1130, 2013.

[55] G. Iosifidis, L. Gao, J. Huang, and L. Tassiulas, "Incentive Mechanisms for User-Provided Networks," IEEE Communications Magazine, vol. 52, no. 9, pp. 20-27, 2014.

[56] _ " "An Iterative Double Auction for Mobile Data Offloading," in Proc. of the 11th Int.l Symposium on Modeling \& Optimization in Mobile, Ad Hoc \& Wireless Networks (WiOpt). IEEE, Tsukuba Science City, Japan, May 2013, pp. 154-161.

[57] X. Wang, M. Chen, T. Taleb, A. Ksentini, and V. Leung, "Cache in the air: exploiting content caching and delivery techniques for $5 \mathrm{G}$ systems," IEEE Communications Magazine, vol. 52, no. 2, pp. 131-139, February 2014.

[58] K. Poularakis, G. Iosifidis, and L. Tassiulas, "Joint caching and base station activation for green heterogeneous cellular networks," in IEEE International Conference on Communications (ICC'15), London, UK, 2015.

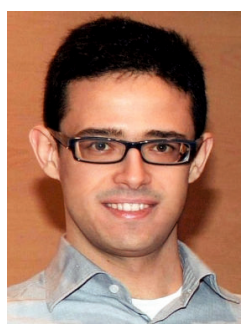

Michele Mangili received his M.S. degree in Computer Science and Engineering from the University of Bergamo in 2012. He is currently pursuing an international Ph.D. in Information Engineering at LRI (Laboratory for Computer Science), Paris-Sud University and DEIB, Politecnico di Milano. His current research interests are Content-Centric Networks, Network Modelling, Planning and Optimization. He is a student member of the IEEE.

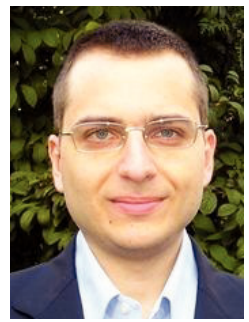

Fabio Martignon received the M.S. and the Ph.D. degrees in telecommunication engineering from the Politecnico di Milano in October 2001 and May 2005 , respectively. He has been associate professor at University of Bergamo, and he is now Full Professor in LRI (Laboratory for Computer Science) at Paris-Sud University, and member of Institut Universitaire de France. His current research activities include cognitive radio networks, content-centric networks, network planning and game theory applications to networking problems. He is a member of

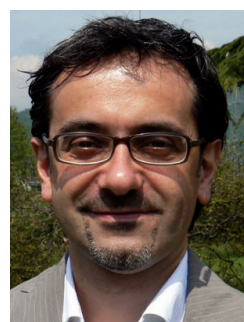

Antonio Capone is Full Professor at the Information, Communication and Bioengineering Technology Department (Dipartimento di Elettronica, Informazione e Bioingegneria) of Politecnico di Milano, where he is the director of the Advanced Network Technologies Laboratory (ANTLab). Dr. Capone is co-founder and CTO of MobiMESH (www.mobimesh.eu), a spin-off company of Politecnico di Milano. His expertise is on networking and his main research activities include protocol design (MAC and routing) and performance evaluation of wireless access and multi-hop networks, traffic management and quality of service issues in IP networks, and network planning and optimization. $\mathrm{He}$ received the M.S. and Ph.D. degrees in electrical engineering from the Politecnico di Milano in 1994 and 1998, respectively. He served as editor of IEEE/ACM Trans. on Networking and he currently serves as editor of IEEE Trans. on Mobile Computing, Wireless Communications and Mobile Computing (Wiley), Computer Networks (Elsevier), and Computer Communications (Elsevier). He is a Senior Member of the IEEE.

the IEEE.

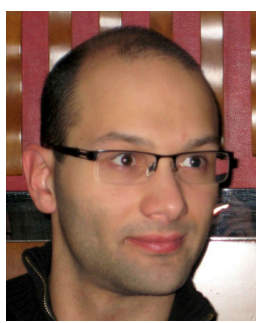

Stefano Paris is Senior Researcher at the Mathematical and Algorithmic Sciences Lab of the France Research Center of Huawei Technologies Co. Ltd. $\mathrm{He}$ has also been working as Assistant Professor at the Department of Computer Science (LIPADE) of Paris Descartes University. He received his M.S. degree in Computer Engineering from University of Bergamo in 2007, and the Ph.D. in Information Engineering from Politecnico di Milano in 2011. His main research interests include topics related to optimization, performance evaluation and security of wireless and wired networks. He is a member of the IEEE. 